\title{
Fatty acids characterization and oxidative stability of spray dried designer egg powder
}

Amna Javed', Muhammad Imran², Nazir Ahmad² and Abdullah ljaz Hussain ${ }^{3}$

\begin{abstract}
Background: Designer eggs (DEs) have gained positive importance in maintaining cholesterol level, triglyceride profile and protection towards cardiovascular diseases due to the presence of essential fatty acids (EFAs) such as omega-3 (or) n-3 fatty acids. However, extreme heat conditions effect the quality as well as quantity of EFAs during the production of designer egg dried powder (DEDP). Therefore, the main mandate of research was the development of DEDP and determination of spray drying conditions impact on fatty acids composition of DEDP samples.

Methods: The DEs were produced, collected, de-shelled, homogenized and diluted before spray drying to get fine powder. The spray drying of DEs was carried out using a laboratory spray drier. An experimental design was used for the drying parameters, where the inlet air temperature was varied $\left(160,180\right.$ and $\left.200^{\circ} \mathrm{C}\right)$, feed flow rate $(200,300$ and $400 \mathrm{~mL} /$ hr), atomization speed $(16,000,20,000$ and $24,000 \mathrm{rpm})$ and outlet air temperature $\left(60,70\right.$ and $\left.80^{\circ} \mathrm{C}\right)$ at different levels. For convenience of experimental design coding was used. The DEDP was collected in a single cyclone separator and was stored after packaging for consecutive 2 months at $25^{\circ} \mathrm{C}$ and $4{ }^{\circ} \mathrm{C}$, respectively. The powder yield was calculated from the collected dry mass in the collecting vessel divided by the processed whole egg diluted matter. The total lipids of DEDP samples were determined gravimetrically. The esters of fatty acids in each sample were prepared and analyzed through Gas Chromatograph apparatus. The oxidative stability of DEDP samples was estimated by following standard procedure of peroxide value.

(Continued on next page)
\end{abstract}

\footnotetext{
* Correspondence: imran@gcuf.edu.pk

${ }^{2}$ Institute of Home and Food Sciences, Faculty of Life Sciences, Government

College University, Faisalabad, Pakistan

Full list of author information is available at the end of the article
} 
(Continued from previous page)

Results: The powder yield of DEDP as a result of different operating conditions was found in the range of $30.06 \pm 0.22 \mathrm{~g} /$ $500 \mathrm{~mL}$ to $62.10 \pm 0.46 \mathrm{~g} / 500 \mathrm{~mL}$ DEs sample. The decreasing trend in moisture content $(4.4 \pm 0.16 \%$ towards $4.0 \pm 0.09 \%)$ and total fat content $(45 \pm 0.65 \mathrm{~g} / 100 \mathrm{~g}$ towards $41 \pm 0.35 \mathrm{~g} / 100 \mathrm{~g}$ ) in DEDP samples was observed with increased inlet and outlet temperature while fat content increased at high feed flow rate and atomization speed. In this study, loss of PUFAs in DEDP samples was followed due to their active role regarding to human health. For alpha-linolenic (ALA) fatty acids, maximum value at $4^{\circ} \mathrm{C}$ observed was $127.32 \pm 0.27 \mathrm{mg} / 50 \mathrm{~g}$ egg and $124.43 \pm 0.32 \mathrm{mg} / 50 \mathrm{~g}$ egg while the minimum value observed for ALA was $100.15 \pm 0.09 \mathrm{mg} / 50 \mathrm{~g}$ egg and $97.15 \pm 0.06 \mathrm{mg} / 50 \mathrm{~g}$ egg after 30 and 60 days storage, respectively. The significant decrease trend for eicosapentaenoic (EPA) fatty acids values from $11.78 \pm 0.31 \mathrm{mg} / 50$ $\mathrm{g}$ egg to $2.18 \pm 0.14 \mathrm{mg} / 50 \mathrm{~g}$ egg at $25^{\circ} \mathrm{C}$ under spray dried conditions of inlet air temperature $\left(180^{\circ} \mathrm{C}\right)$, feed flow rate $(300 \mathrm{~mL} / \mathrm{hr})$, atomization speed $(24,000 \mathrm{rpm})$ and outlet air temperature $\left(80^{\circ} \mathrm{C}\right)$ after 60 days storage period was noted. The docosahexaenoic (DHA) fatty acids value in DEDP was decreased from $15.49 \pm 0.79 \mathrm{mg} / 50 \mathrm{~g}$ egg (0 day) to $10.10 \pm 0.64 \mathrm{mg} / 50 \mathrm{~g}$ egg at 60 days $\left(4^{\circ} \mathrm{C}\right)$ and same decreasing trend was observed at $25^{\circ} \mathrm{C}$. The decreasing order for total omega-3 fatty acids retention in DEDP during storage intervals was found as $162.33 \pm 1.64 \mathrm{mg} / 50 \mathrm{~g}$ egg $>158.61 \pm 1.53 \mathrm{mg} / 50 \mathrm{~g}$ egg $>148.03 \pm 1.57 \mathrm{mg} / 50 \mathrm{~g}$ egg $\left(0,30\right.$ and 60 days stored at $\left.4^{\circ} \mathrm{C}\right)$ and $162.33 \pm 1.64 \mathrm{mg} / 50 \mathrm{~g}$ egg $>151.56 \pm 1.54 \mathrm{mg} / 50 \mathrm{~g}$ egg $>135.89 \pm 1.62 \mathrm{mg} / 50 \mathrm{~g}$ egg $\left(0,30\right.$ and 60 days stored at $\left.25^{\circ} \mathrm{C}\right)$. The peroxide value (PV) levels obtained in DEDP samples after 60 days were higher $\left(0.78 \pm 0.06,0.81 \pm 0.02 \mathrm{meq} / \mathrm{kg} \mathrm{O}_{2}\right)$ when compared to initial readings at 0 day $(0.65 \pm 0.04 \mathrm{meq} / \mathrm{kg} \mathrm{O}$ ). The PV of DEDP samples reached their maximum peaks after 60 days at $25^{\circ} \mathrm{C}$. The increasing order showed that lipid oxidation increased with storage. However, the overall PV never exceeded the limit of $10(\mathrm{meq} / \mathrm{kg})$ considered as a threshold limit.

Conclusions: Extreme hot conditions $\left(>180^{\circ} \mathrm{C}\right.$ ) of spray dryer reduce the quality of designer egg dry powder. Extreme conditions assist PUFAs loss and decrease in storage stability due to high lipid oxidation.

Keywords: Lipid oxidation, Storage stability, Fatty acids profile, Omega eggs, Spray drying,

\section{Background}

Lipids are considered an important component of food as well as most biological systems. Mostly saturated and monounsaturated fatty acids biosynthesized in the body, but polyunsaturated fatty acids (PUFAs) must be provided through the diet or other sources for maintenance of health $[1,2]$. PUFAs poses biological and medicinal interest due to multiple beneficial effects on health, including anti-inflammatory, cardioprotective and anticancer activities etc. [3-5]. The designer eggs (DEs) are widely used regarding to human health in providing various essential fatty acids (EFAs) such as omega-3 (or) n-3 fatty acids; Alpha-Linolenic acid (ALA): $\mathrm{C}_{18: 3 n-3}$, Eicosapentaenoic acid (EPA): $\mathrm{C}_{20: 5 n-3}$ and Docosahexaenoic acid (DHA): $\mathrm{C}_{22: 6 n-3}$. DEs show beneficial effects regarding to improve the blood concentration of omega- 3 fatty acids and high-density lipoproteins [6].

However, lipid oxidation negatively affects the integrity of biological systems and causes quality deterioration in food. The oxidative instability possesses objectionable off-flavors, loss of nutrients and bioactives that leads to formation of potentially toxic compounds, thus making the lipid or lipid containing foods unsuitable for human health [7]. Destructive irreversible cellular and tissue effects, pathophysiology of numerous diseases and variety of health conditions including inflammation, mutagenesis, atherosclerosis, carcinogenesis and aging process are associated with fatty acid oxidation products in human foods [8-10].
Spray drying is a suspended-particle technology which has a wide range of applications in mostly food, pharmaceutical and biotech industry. In spray drying process, a liquid droplet is rapidly dried, when it comes into contact with a stream of hot air (temperature range from 100 to $300{ }^{\circ} \mathrm{C}$ ) and convert it into powder form [11]. Spray drying produces powder with good handling, easiness in transportation and highly functional in nature [12-16]. Moreover, dried egg and egg products drive the product manufacture's attention for ready to use in baked, soups and meat products. The spray dried egg powder has been suggested to be the easily digestible and good source of nutrients from egg products [17]. A comprehensive literature search reported that no significant research has been done on the optimization of the spray drying parameters to produce highest quality designer egg dried powder (DEDP). The present study was undertaken to optimize the spray drying process under different ranges of inlet air temperature, feed flow rate, atomization speed and outlet air temperature to have maximum retention of PUFAs at the different storage periods and temperatures.

\section{Methods}

Raw materials

The raw materials such as chia seed (Salvia hispanica L.) and other cereal grains were procured from grains commercial market, Punjab, Pakistan. The seeds were 
cleaned to remove any debris or field dirt and any other extraneous matters. The menhaden fish oil was obtained from commercial fish processing industry, Punjab, Pakistan.

\section{Diet composition and feeding trial}

The feeding trial was conducted on medium-heavy Leghorn layers (20 weeks old; uniform weight) in wire-mesh pens of commercial poultry house, Punjab, Pakistan. The birds were used to keep in $17 \mathrm{~h}$ light and $7 \mathrm{~h}$ dark day. All hens were fed on control diet from 20th week of their age before the trial which was helpful for baseline data. The temperature $25 \pm 2{ }^{\circ} \mathrm{C}$ and humidity $70 \pm 5 \%$ remained constant throughout the eight experimental weeks. The Leghorn layers were randomly distributed into control and designer feed treatments of 40 layers each. Each bird activity was observed on daily basis. Routine vaccination and medication were conducted as management suggested. The feed ingredient profile for control and designer eggs production has been presented in Table 1 . The mixed crumble feed was produced weekly and packed in air tight feed bins to avoid oxidation and moisture build up and placed in dark cooled room to minimize the exposure to environment. The DEs were produced and collected after 8 weeks of feeding trial.

\section{Sample preparation}

The DEs $(n=600)$ were cautiously de-shelled and whole egg liquid $(n=20)$ for each treatment was collected in a graduated cylinder. Water was added to the whole egg liquid (60\% protein: $40 \%$ yolk) and mixed well to make a fine dilution. The concentration of this continuous dilution was 1:1 ratio. Thereafter, homogenization of egg sample was carried out using a homogenizer. A sifting was conducted to eliminate the chalazas and the suspended matter. The whole egg sample was diluted before spray drying to get fine powder [18].

\section{Spray drying procedure}

A laboratory spray drier No. 1 (Anhydro A/S, Ostmarken 8, DK-2860 Soborg, Copenhagen, Denmark) was used in this study. The schematic diagram of the lab-scale spray dryer is demonstrated in Fig. 1. The internal diameter of representative spray dryer was $1.0 \mathrm{~m}$ and $2.6 \mathrm{~m}$ were height. The upper cylindrical portion of the unit is $1.3 \mathrm{~m}$ in height, and the lower conical section has a height of $1.3 \mathrm{~m}$. The maximum inlet and outlet temperatures are 300 and $90{ }^{\circ} \mathrm{C}$, respectively. Maximum atomizer speed is $50,000 \mathrm{rpm}$ which is obtained by the power supply $0.736 \mathrm{~kW}$ electric motor. Air is heated by air heater using a power of $9 \mathrm{~kW}$ with compressed air consumption of $120 \mathrm{l} / \mathrm{min}$ and compressed air pressure of $4 \mathrm{~kg} / \mathrm{cm}^{2}$ [19]. An experimental design was used for the drying parameters, where the inlet air temperature was varied $\left(160,180\right.$ and $\left.200^{\circ} \mathrm{C}\right)$, feed flow rate $(200$, 300 and $400 \mathrm{~mL} / \mathrm{hr})$, atomization speed $(16,000,20,000$ and $24,000 \mathrm{rpm})$ and outlet air temperature $(60,70$ and $80^{\circ} \mathrm{C}$ ) at different levels. For convenience of experimental design coding was used which is presented in Table 2 . The designer egg dried powder (DEDP) from each treatment $(500 \mathrm{~mL})$ was collected in a single cyclone separator and was stored at $25^{\circ} \mathrm{C}$ and $4{ }^{\circ} \mathrm{C}$, respectively after packaging for consecutive 2 months.

\section{Powder yield, total fat and fatty acids composition of DEDP}

The powder yield was calculated from the collected dry mass in the collecting vessel divided by the processed whole egg diluted matter. The total lipids of DEDP samples were determined gravimetrically according to the AOAC [20] Method No. 923.07. The esters of fatty acids in each sample were prepared and analyzed through Gas Chromatograph apparatus equipped with an auto sampler, flame-ionization detector (FID) and supelco wax column $(30 \mathrm{~m} \times 0.25 \mu \mathrm{m}$ film coating) according to AOCS [21]. Briefly, transferred $1 \mathrm{~g}$ DEDP sample to the screw capped tube $(16$ X $150 \mathrm{~mm})$. Added $10 \mathrm{~mL}$ hexane containing 0.1\% BHT (an antioxidant to help prevent the peroxidation of fatty acid containing double bonds). Caped the tube tightly and shaked vigorously for $1 \mathrm{~min}$. Then put in ultrasonic water bath for $5 \mathrm{~min}$. Centrifuged the tube at $1500 \mathrm{X} \mathrm{g}$ for $5 \mathrm{~min}$. Put up the experimental tubes into a heating block heated to $60^{\circ} \mathrm{C}$ and a stream

Table 1 Feed ingredient profile for control and designer eggs production

\begin{tabular}{|c|c|c|c|c|c|c|c|c|c|c|c|c|c|}
\hline \multirow[t]{2}{*}{ Treatment } & \multicolumn{13}{|c|}{ Feed Ingredients $(\mathrm{g} / 100 \mathrm{~g})^{\mathrm{b}}$} \\
\hline & Corn & Wheat & $\begin{array}{l}\text { Rice } \\
\text { polishing }\end{array}$ & $\begin{array}{l}\text { Canola } \\
\text { meal }\end{array}$ & Flaxseed & $\begin{array}{l}\text { Chia } \\
\text { seed }\end{array}$ & $\begin{array}{l}\text { Fish } \\
\text { oil }\end{array}$ & $\begin{array}{l}\text { Gluten } \\
(60 \%)\end{array}$ & $\begin{array}{l}\text { Soybean } \\
\text { meal }\end{array}$ & $\begin{array}{l}\text { Vegetable } \\
\text { oil }\end{array}$ & $\begin{array}{l}\text { Dicalcium } \\
\text { phosphate }\end{array}$ & $\begin{array}{l}\text { Lime stone } \\
\text { (ground) }\end{array}$ & $\begin{array}{l}\text { Vitamin/ } \\
\text { mineral } \\
\text { premix }\end{array}$ \\
\hline $\begin{array}{l}\text { Control } \\
\text { feed }^{c}\end{array}$ & 35 & 5 & 15 & 15 & - & - & - & 5 & 8 & 8 & 1.5 & 7 & 0.5 \\
\hline $\begin{array}{l}\text { Designer } \\
\text { feed }^{c}\end{array}$ & 35 & 10 & 8 & 9 & 5 & 10 & 1 & 3 & 8 & 2 & 1.5 & 7 & 0.5 \\
\hline
\end{tabular}

${ }^{\mathrm{a}}$ Cottonseed oil

${ }^{b}$ Crumble form of feed

Isocaloric feeds 


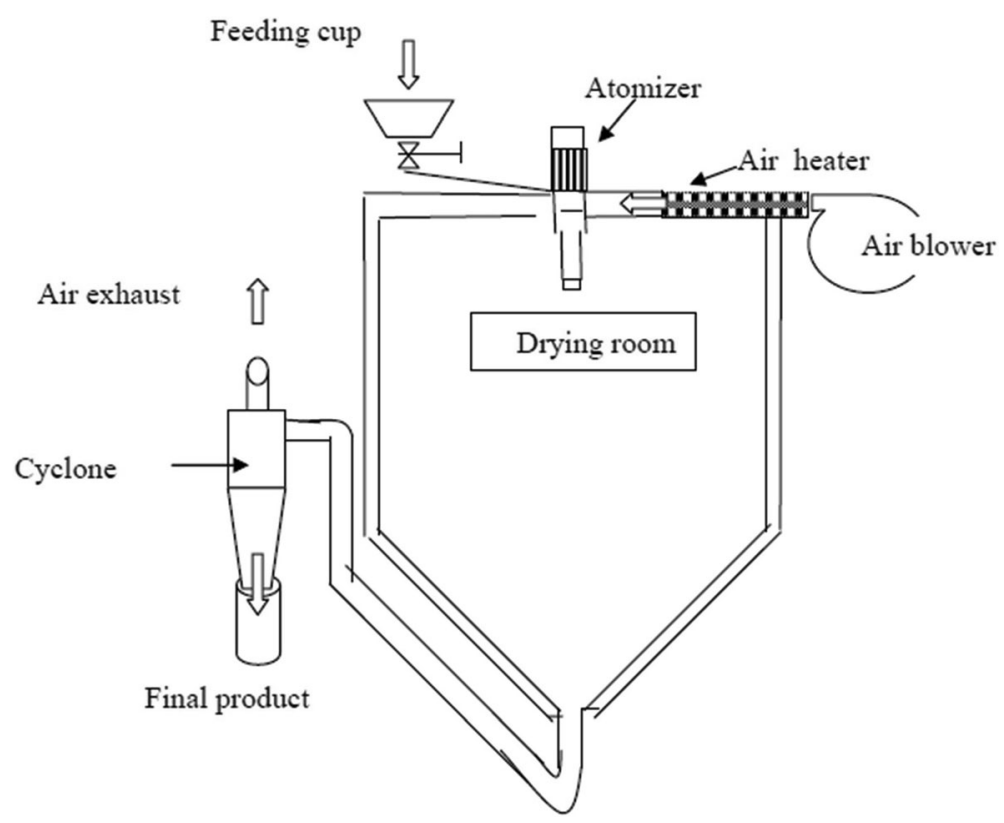

Fig. 1 The schematic diagram of the lab-scale spray dryer

of nitrogen gas was blown into the tube to facilitate evaporation of the hexane. Toluene $(1 \mathrm{~mL})$ was added to $50 \mathrm{mg}$ of sample in a screw top test tube. Subsequently, $2 \mathrm{~mL}$ of boron trichloride-methanol solution was added and the mixture was flushed with nitrogen gas for $10 \mathrm{~s}$ and heated in a water bath at $60^{\circ} \mathrm{C}$ for $10 \mathrm{~min}$. Once cooled, water $(2 \mathrm{~mL})$ and hexane $(2 \mathrm{~mL})$ were added into the test tube and shaken lightly to extract the fatty acid methyl esters (FAMEs). Anhydrous sodium sulfate was added to the hexane extracts to remove moisture then the anhydrous hexane extracts were transferred into a $10 \mathrm{~mL}$ volumetric flask and filled to volume with hexane. The moisture removal step was carried out twice to ensure maximum extraction of FAMEs from the oil. FAMEs were then analyzed by gas chromatography. The FAMEs samples $(1 \mu \mathrm{L})$ were injected with Helium (1 $\mathrm{mL} / \mathrm{min}$ ) as a carrier gas onto the column, which was programmed for operating conditions such as column oven temperature $160^{\circ} \mathrm{C} @ 0$ min with subsequent increase of $3{ }^{\circ} \mathrm{C} / \mathrm{min}$ until $180^{\circ} \mathrm{C}$. The column oven

Table 2 Coded and actual levels of independent variables for optimization of response factors as determined by Box-Behnken design

\begin{tabular}{lllll}
\hline Independent variables & Units & \multicolumn{3}{l}{ Coded levels } \\
\cline { 3 - 5 } & & -1 & 0 & +1 \\
\hline Inlet air temperature & ${ }^{\circ} \mathrm{C}$ & 160 & 180 & 200 \\
Feed flow rate & $\mathrm{mL} / \mathrm{hr}$ & 200 & 300 & 400 \\
Atomization speed & $\mathrm{rpm}$ & 16,000 & 20,000 & 24,000 \\
Outlet air temperature & ${ }^{\circ} \mathrm{C}$ & 60 & 70 & 80 \\
\hline
\end{tabular}

temperature was increased from $180^{\circ} \mathrm{C}$ to $220^{\circ} \mathrm{C} @ 1^{\circ}$ $\mathrm{C} / \mathrm{min}$ and was held for $7.5 \mathrm{~min}$ at $220^{\circ} \mathrm{C}$. Split ratio was $50 \%$ with injector $240{ }^{\circ} \mathrm{C}$ and detector $250^{\circ} \mathrm{C}$ temperatures. The peak areas and total fatty acids composition were calculated for each sample by retention time using Varian Chem Station software.

\section{Peroxide value of DEDP}

The peroxide value of DEDP samples was estimated by following the AOCS standard procedure (Method No. Cd 8-53) [21].

\section{Statistical analysis}

The analysis of experiments was carried out according to Montgomery [22]. Each experiment was performed in triplicate and the average values were taken as response. The significance of all terms was analyzed statistically by computing mean square at probability $(\mathrm{p})$ of 0.05 using MATLAB $^{\circ}$ (Ver. 7.9.0) software (Mathworks, Inc., Natick, USA).

\section{Results and discussion}

The fatty acids analysis of poultry control and designer feed has been documented in Table 3. In this study, the effects of spray-drying conditions were majorly investigated (Inlet and outlet air temperatures, feed flow rate and atomization pressure) on powder yield along with retention of PUFAs. The powder yield of DEDP as a result of different operating conditions was found in the range of $30.06 \pm 0.22 \mathrm{~g} / 500 \mathrm{~mL}$ to $62.10 \pm 0.46 \mathrm{~g} / 500 \mathrm{~mL}$ DEs samples (Fig. 2). The inlet temperature, outlet 
Table 3 Fatty acids analysis of poultry control and designer feed

\begin{tabular}{lclllllll}
\hline Treatment & \multicolumn{1}{l}{ Fatty acids $\left(\%\right.$ of TFA ${ }^{\mathrm{a}}$ ) } & \multicolumn{1}{l}{} & & \\
\cline { 2 - 9 } & Palmitic & Stearic & Oleic & Linoleic & Linolenic & Arachidonic & Eicosapentaenoic & Docosahexaenoic \\
\hline Control feed & 12.76 & 15.48 & 28.33 & 37.57 & 2.41 & 0.5 & 0.34 & 0.06 \\
Designer feed & 8.33 & 10.89 & 23.12 & 39.45 & 10.64 & 0.25 & 1.44 & 0.72 \\
\hline
\end{tabular}

${ }^{\text {a }}$ Total fatty acids

temperature and the atomization speed were the most major factors affecting the powder yield of DEDP. The results showed that the powder yield decreased with increasing inlet temperature, outlet temperature and the atomization speed. The optimized conditions of inlet air temperature $\left(198-199^{\circ} \mathrm{C}\right)$, feed flow rate $(398-399 \mathrm{~mL} /$ $\mathrm{hr})$, atomization speed $(16000-16,010 \mathrm{rpm})$ and outlet air temperature $\left(76-77^{\circ} \mathrm{C}\right)$ were found for maximum yield of DEDP samples $(66.20 \pm 0.20 \mathrm{~g} / 500 \mathrm{~mL})$.

The spray drying variables caused substantial changes in the whole egg powder yield shown by the previous studies. Same results concluded by Bahnasawy et al. [19] that the powder yield decreased slightly with increasing both atomization speed and drying temperature for all blends under study. These results may be due to production of DEDP with fine particles structure at higher temperature and speed conditions and these conditions force the very fine particles to go out with exhaust air. Atomizer is the heart of spray drying process that disperses material into precise particles so that surface area of the liquid material is increased. In this way, material is dispersed well within the dryer chamber. After atomization, the droplets produced should not be very huge as that condition developed partially dried powder and even nor so tiny in size or structure as that leads to difficulty in recovery of DEDP samples. The final shape and kind of dried powder product depends on the chemical and physical properties of the liquid material, dryer design and operative parameters $[11,23]$.

The trend in decrease of moisture content was observed with increase in conditions. The decreasing trend in moisture content was found from $4.4 \pm 0.16 \%$ (highest value) towards $4.0 \pm 0.09 \%$ (lowest value) in DEDP samples with changes in operating conditions especially inlet and outlet temperature. At the same time increased moisture content was detected at high feed flow rate. The data trend also showed that moisture content was inversely proportional to atomization speed of spray dryer. In a similar way, the total fat content decreased from $45 \pm 0.65 \mathrm{~g} / 100 \mathrm{~g}$ (highest value) to $41 \pm 0.35 \mathrm{~g} / 100$ $\mathrm{g}$ (lowest value) in DEDP samples with increased inlet and outlet temperature while fat content increased at high feed flow rate and atomization speed.

The normal eggs possessed the ALA $(0.78 \pm 0.14$ $\mathrm{mg} / 50 \mathrm{~g}$ egg), EPA $(0.11 \pm 0.06 \mathrm{mg} / 50 \mathrm{~g}$ egg $)$, DHA $\left(0.14 \pm 0.07 \mathrm{mg} / 50 \mathrm{~g}\right.$ egg) and PV $\left(0.324 \mathrm{meq} / \mathrm{kg} \mathrm{O}_{2}\right)$,

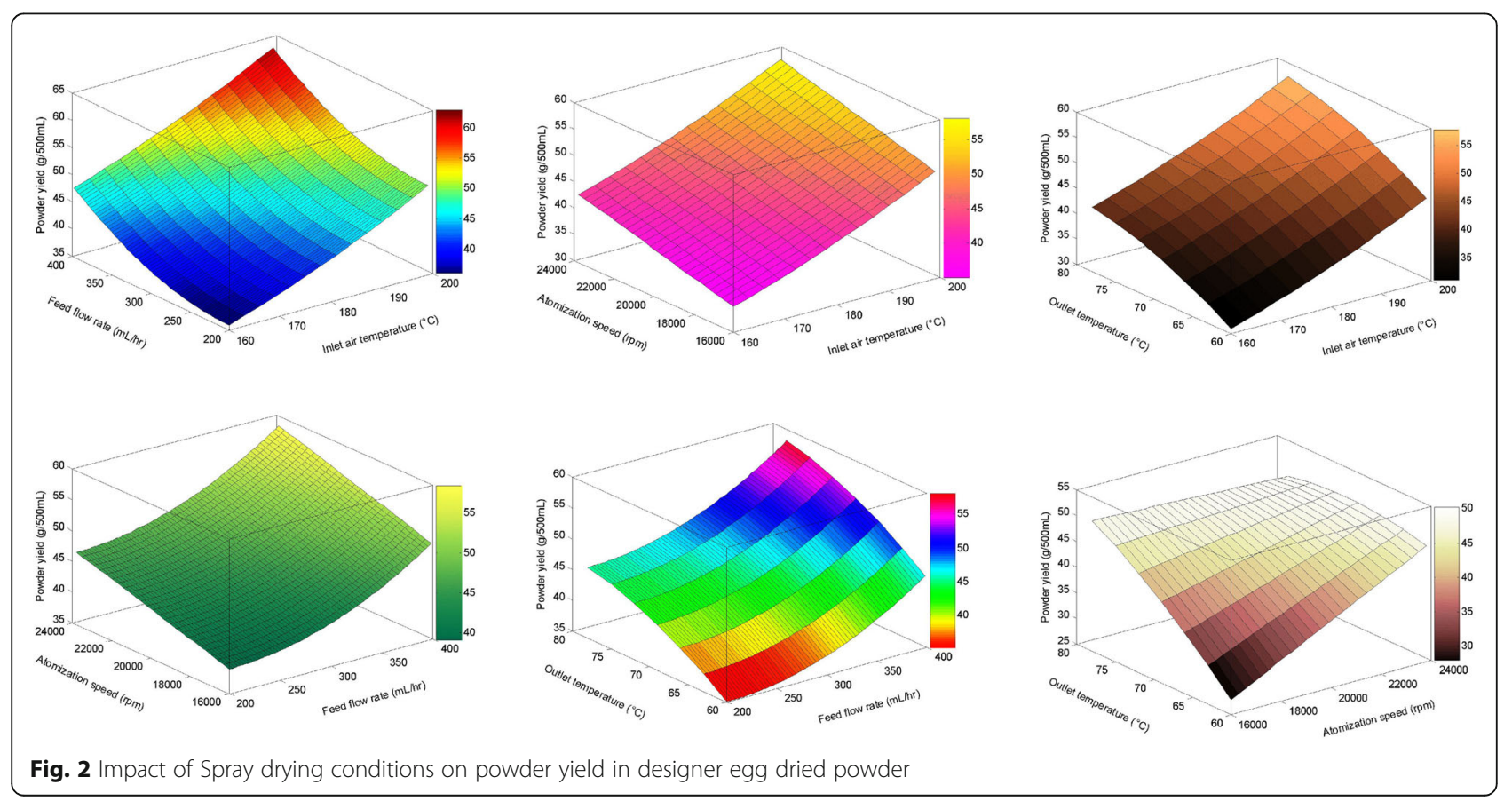


respectively. Whereas, the designer eggs before spray drying process contained ALA (130.23 $\pm 0.28 \mathrm{mg} / 50 \mathrm{~g}$ egg), EPA $(15.10 \pm 0.37 \mathrm{mg} / 50 \mathrm{~g}$ egg), DHA $(20.17 \pm 0.67 \mathrm{mg} / 50 \mathrm{~g}$ egg), total omega-3 fatty acids $(165.50 \pm 2.21 \mathrm{mg} / 50 \mathrm{~g}$ egg $)$ and PV $(0.418 \mathrm{meq} / \mathrm{kg} \mathrm{O}$ ), respectively. In this study, loss of PUFAs was followed due to their active role regarding to human health.

To check the reliability of fatty acid retention in DEDP, it was determined in 29 DEDP samples for 30 and 60 storage days at two different temperatures likewise $4{ }^{\circ} \mathrm{C}$ and $25^{\circ} \mathrm{C}$, respectively. The results demonstrated that the contents of alpha-linolenic fatty acids were not stable under variable storage intervals at different conditions (Table 4). The inlet air temperature and outlet air temperature were seen to be as major factors affecting the essential fatty acids content in samples. The alpha-linolenic acid, eicosapentaenoic and docosahexaenoic fatty acids contents decreased significantly on storage at higher temperature as compared to lower temperature under different conditions. For alpha-linolenic fatty acids, maximum value at $4{ }^{\circ} \mathrm{C}$ observed

Table 4 Impact of Spray drying conditions on alpha-linolenic fatty acids retention in designer egg dried powder at different days and storage intervals

\begin{tabular}{|c|c|c|c|c|c|c|c|c|c|}
\hline \multirow{3}{*}{$\begin{array}{l}\text { Spray } \\
\text { dryer } \\
\text { process } \\
\text { run }\end{array}$} & \multicolumn{4}{|c|}{ Independent variables } & \multicolumn{5}{|c|}{ ALA (mg/50 g egg) } \\
\hline & \multirow{2}{*}{$\begin{array}{l}\text { Inlet air } \\
\text { temperature } \\
\left({ }^{\circ} \mathrm{C}\right) \\
\end{array}$} & \multirow{2}{*}{$\begin{array}{l}\text { Feed flow } \\
\text { rate } \\
(\mathrm{mL} / \mathrm{hr})\end{array}$} & \multirow{2}{*}{$\begin{array}{l}\text { Atomization } \\
\text { speed (rpm) }\end{array}$} & \multirow{2}{*}{$\begin{array}{l}\text { Outlet } \\
\text { temperature } \\
\left({ }^{\circ} \mathrm{C}\right)\end{array}$} & \multirow[t]{2}{*}{ O Day } & \multicolumn{2}{|c|}{ Storage at Temperature $4^{\circ} \mathrm{C}$} & \multicolumn{2}{|c|}{ Storage at Temperature $25^{\circ} \mathrm{C}$} \\
\hline & & & & & & 30 Days & 60 Days & 30 Days & 60 Days \\
\hline 1 & $160(-1)$ & $300(0)$ & $16,000(-1)$ & $70(0)$ & $127.57 \pm 0.46^{\mathrm{ab}}$ & $126.22 \pm 0.41^{b}$ & $123.05 \pm 0.44^{\mathrm{cd}}$ & $123.12 \pm 0.42^{\mathrm{cd}}$ & $118.52 \pm 0.41^{\mathrm{ef}}$ \\
\hline 2 & $180(0)$ & $200(-1)$ & $20,000(0)$ & $60(-1)$ & $118.81 \pm 0.70^{\mathrm{ef}}$ & $117.34 \pm 0.62^{f}$ & $114.33 \pm 0.61^{g h}$ & $114.25 \pm 0.62^{g h}$ & $109.55 \pm 0.67^{j}$ \\
\hline 3 & $180(0)$ & $300(0)$ & $16,000(-1)$ & $80(+1)$ & $116.58 \pm 0.47^{f g}$ & $117.19 \pm 0.44^{f}$ & $114.14 \pm 0.47^{g h}$ & $112.37 \pm 0.41^{\mathrm{hi}}$ & $107.53 \pm 0.44^{k}$ \\
\hline 4 & $180(0)$ & $400(+1)$ & $20,000(0)$ & $60(-1)$ & $125.67 \pm 0.54^{\mathrm{bc}}$ & $124.05 \pm 0.44^{c}$ & $121.76 \pm 0.62^{d}$ & $121.53 \pm 0.42^{d}$ & $116.43 \pm 0.31^{\mathrm{fg}}$ \\
\hline 5 & $180(0)$ & $200(-1)$ & $16,000(-1)$ & $70(0)$ & $118.30 \pm 0.19^{e f}$ & $117.04 \pm 0.13^{f}$ & $114.75 \pm 0.15^{g h}$ & $114.21 \pm 0.14^{\mathrm{gh}}$ & $109.51 \pm 0.16^{j}$ \\
\hline 6 & $160(-1)$ & $300(0)$ & $24,000(+1)$ & $70(0)$ & $114.75 \pm 0.61^{g h}$ & $113.66 \pm 0.51^{\mathrm{h}}$ & $110.45 \pm 0.34^{\mathrm{ij}}$ & $110.55 \pm 0.43^{\mathrm{ij}}$ & $105.87 \pm 0.75^{1}$ \\
\hline $7\left(C_{1}\right)$ & $180(0)$ & $300(0)$ & $20,000(0)$ & $70(0)$ & $114.82 \pm 0.70^{g h}$ & $113.55 \pm 0.45^{h}$ & $110.76 \pm 0.61^{i j}$ & $110.72 \pm 0.67^{\mathrm{ij}}$ & $105.01 \pm 0.52^{1}$ \\
\hline $8\left(C_{2}\right)$ & $180(0)$ & $300(0)$ & $20,000(0)$ & $70(0)$ & $114.68 \pm 0.54^{g h}$ & $113.35 \pm 0.24^{h}$ & $110.41 \pm 0.32^{i j}$ & $110.42 \pm 0.31^{i j}$ & $105.42 \pm 0.34^{\prime}$ \\
\hline 9 & $180(0)$ & $300(0)$ & $24,000(+1)$ & $60(-1)$ & $115.97 \pm 0.87^{9}$ & $114.72 \pm 0.61^{g h}$ & $111.95 \pm 0.82^{i}$ & $111.32 \pm 0.24^{i}$ & $106.45 \pm 0.35^{\mathrm{kl}}$ \\
\hline 10 & $200(+1)$ & $300(0)$ & $24,000(+1)$ & $70(0)$ & $101.19 \pm 0.12^{n}$ & $100.15 \pm 0.09^{\text {no }}$ & $97.15 \pm 0.06^{\mathrm{P}}$ & $97.55 \pm 0.08^{p}$ & $92.68 \pm 0.13^{5}$ \\
\hline $11\left(C_{3}\right)$ & $180(0)$ & $300(0)$ & $20,000(0)$ & $70(0)$ & $114.76 \pm 0.69^{g h}$ & $113.65 \pm 0.53^{h}$ & $110.22 \pm 0.54^{i j}$ & $110.01 \pm 0.51^{\mathrm{ij}}$ & $105.03 \pm 0.42^{1}$ \\
\hline $12\left(C_{4}\right)$ & $180(0)$ & $300(0)$ & $20,000(0)$ & $70(0)$ & $114.75 \pm 0.63^{g h}$ & $113.64 \pm 0.57^{h}$ & $110.33 \pm 0.64^{i j}$ & $110.43 \pm 0.37^{\mathrm{ij}}$ & $105.56 \pm 0.46^{1}$ \\
\hline 13 & $180(0)$ & $200(-1)$ & $20,000(0)$ & $80(+1)$ & $106.03 \pm 0.31^{\mathrm{kl}}$ & $105.44 \pm 0.32^{1}$ & $102.55 \pm 0.40^{\mathrm{mn}}$ & $102.21 \pm 0.22^{\mathrm{mn}}$ & $97.56 \pm 0.42^{p}$ \\
\hline 14 & $200(+1)$ & $400(+1)$ & $20,000(0)$ & $70(0)$ & $111.19 \pm 0.49^{i}$ & $110.05 \pm 0.43^{i j}$ & $107.61 \pm 0.53^{k}$ & $107.31 \pm 0.28^{k}$ & $102.61 \pm 0.52^{\mathrm{mn}}$ \\
\hline 15 & $160(-1)$ & $400(+1)$ & $20,000(0)$ & $70(0)$ & $124.61 \pm 0.54^{c}$ & $123.55 \pm 0.41^{c d}$ & $120.75 \pm 0.65^{\mathrm{de}}$ & $120.62 \pm 0.56^{\mathrm{de}}$ & $115.45 \pm 0.38^{9}$ \\
\hline 16 & $180(0)$ & $400(+1)$ & $20,000(0)$ & $80(+1)$ & $113.95 \pm 0.84^{h}$ & $112.65 \pm 0.54^{\mathrm{hi}}$ & $109.74 \pm 0.61^{j}$ & $109.32 \pm 0.57^{j}$ & $104.33 \pm 0.67^{1 \mathrm{~m}}$ \\
\hline 17 & $160(-1)$ & $300(0)$ & $20,000(0)$ & $80(+1)$ & $115.57 \pm 0.43^{9}$ & $114.12 \pm 0.35^{g h}$ & $111.32 \pm 0.28^{i}$ & $111.46 \pm 0.37^{i}$ & $106.78 \pm 0.63^{\mathrm{kl}}$ \\
\hline 18 & $200(+1)$ & $300(0)$ & $20,000(0)$ & $80(+1)$ & $102.68 \pm 0.63^{\mathrm{mn}}$ & $101.62 \pm 0.52^{n}$ & $98.33 \pm 0.29^{\circ p}$ & $98.63 \pm 0.50^{\mathrm{op}}$ & $93.98 \pm 0.47^{r}$ \\
\hline 19 & $160(-1)$ & $200(-1)$ & $20,000(0)$ & $70(0)$ & $117.87 \pm 0.71^{f}$ & $116.11 \pm 0.64^{f g}$ & $113.55 \pm 0.42^{h}$ & $113.15 \pm 0.38^{h}$ & $108.09 \pm 0.42^{\mathrm{jk}}$ \\
\hline 20 & $180(0)$ & $400(+1)$ & $16,000(-1)$ & $70(0)$ & $125.70 \pm 0.62^{b c}$ & $124.62 \pm 0.51^{c}$ & $121.32 \pm 0.46^{d}$ & $121.43 \pm 0.32^{d}$ & $116.35 \pm 0.27^{\mathrm{fg}}$ \\
\hline 21 & $180(0)$ & $300(0)$ & $24,000(+1)$ & $80(+1)$ & $103.92 \pm 0.74^{m}$ & $102.01 \pm 0.61^{\mathrm{mn}}$ & $99.73 \pm 0.66^{\circ}$ & $99.59 \pm 0.46^{\circ}$ & $94.67 \pm 0.54^{\mathrm{qr}}$ \\
\hline 22 & $180(0)$ & $300(0)$ & $16,000(-1)$ & $60(-1)$ & $128.37 \pm 0.28^{a}$ & $127.32 \pm 0.27^{\mathrm{ab}}$ & $124.43 \pm 0.32^{c}$ & $124.43 \pm 0.31^{c}$ & $119.87 \pm 0.41^{e}$ \\
\hline 23 & $180(0)$ & $400(+1)$ & $24,000(+1)$ & $70(0)$ & $112.25 \pm 0.72^{\mathrm{hi}}$ & $111.95 \pm 0.84^{i}$ & $108.11 \pm 0.65^{\mathrm{jk}}$ & $108.55 \pm 0.47^{\mathrm{jk}}$ & $103.34 \pm 0.59^{\mathrm{m}}$ \\
\hline 24 & (1) & $300(0)$ & $20,000(0)$ & $60(-1)$ & $127.47 \pm 0.38^{\mathrm{ab}}$ & $126.33 \pm 0.24^{b}$ & $123.64 \pm 0.45^{c d}$ & $123.78 \pm 0.33^{\mathrm{cd}}$ & $118.75 \pm 0.42^{\mathrm{ef}}$ \\
\hline $25\left(C_{5}\right)$ & $180(0)$ & $300(0)$ & $20,000(0)$ & $70(0)$ & $114.13 \pm 0.20^{g h}$ & $113.01 \pm 0.24^{h}$ & $110.01 \pm 0.23^{i j}$ & $110.54 \pm 0.37^{i j}$ & $105.34 \pm 0.29^{\prime}$ \\
\hline 26 & $200(+1)$ & $300(0)$ & $16,000(-1)$ & $70(0)$ & $114.88 \pm 0.51^{g h}$ & $113.22 \pm 0.44^{h}$ & $110.21 \pm 0.33^{\mathrm{ij}}$ & $110.43 \pm 0.36^{\mathrm{ij}}$ & $105.33 \pm 0.21^{\prime}$ \\
\hline 27 & $180(0)$ & $200(-1)$ & $24,000(+1)$ & $70(0)$ & $105.01 \pm 0.22^{1}$ & $104.00 \pm 0.21^{1 \mathrm{~m}}$ & $101.04 \pm 0.32^{n}$ & $101.34 \pm 0.29^{n}$ & $96.34 \pm 0.23^{p q}$ \\
\hline 28 & $200(+1)$ & $200(-1)$ & $20,000(0)$ & $70(0)$ & $104.82 \pm 0.56^{\mathrm{Im}}$ & $103.54 \pm 0.47^{\mathrm{m}}$ & $100.23 \pm 0.51^{\text {no }}$ & $100.32 \pm 0.57^{n o}$ & $95.96 \pm 0.63^{9}$ \\
\hline 29 & $200(+1)$ & $300(0)$ & $20,000(0)$ & $60(-1)$ & $114.19 \pm 0.12^{g h}$ & $113.12 \pm 0.15^{h}$ & $110.21 \pm 0.16^{i j}$ & $110.65 \pm 0.25^{\mathrm{ij}}$ & $105.33 \pm 0.29^{\prime}$ \\
\hline
\end{tabular}

$\mathrm{C1}, \mathrm{C2}, \mathrm{C3}, \mathrm{C4}, \mathrm{C5}$ represent spraying drying process at center points

Experimental model $=$ Box-Behnken design

Total number of spray drying treatments $=29$

No of replicates $=03$

${ }^{a-s}$ values with similar letters show homogenous group within row and column $(p>0.05)$ 
was $127.32 \pm 0.27 \mathrm{mg} / 50 \mathrm{~g}$ egg and $124.43 \pm 0.32 \mathrm{mg} / 50 \mathrm{~g}$ egg (spray drying run 22) while the minimum value observed for ALA was $100.15 \pm 0.09 \mathrm{mg} / 50 \mathrm{~g}$ egg and $97.15 \pm$ $0.06 \mathrm{mg} / 50 \mathrm{~g}$ egg after 30 and 60 days, respectively. The changes calculated for ALA was $21.98 \%$ (at $4{ }^{\circ} \mathrm{C}$ after 30 days), $24.32 \%$ (at $4{ }^{\circ} \mathrm{C}$ after 60 days), $24.01 \%$ (at $25^{\circ} \mathrm{C}$ after 30 days) and $27.80 \%$ (at $25^{\circ} \mathrm{C}$ after 60 days), respectively.

The trend for effects of various storage time intervals for eicosapentaenoic fatty acids is shown in Table 5 . The minimum value for EPA observed was $10.02 \pm 0.21 \mathrm{mg} /$ $50 \mathrm{~g}$ egg under four factors of spray drier i.e. inlet air temperature $\left(200{ }^{\circ} \mathrm{C}\right)$, feed flow rate $(300 \mathrm{~mL} / \mathrm{hr})$, atomization speed $(20,000 \mathrm{rpm})$, outlet air temperature $\left(80{ }^{\circ} \mathrm{C}\right)$ stored at $4{ }^{\circ} \mathrm{C}$ after 30 days of storage. The EPA trend showed that significant decrease $11.78 \pm 0.31 \mathrm{mg} /$ $50 \mathrm{~g}$ egg to $2.18 \pm 0.14 \mathrm{mg} / 50 \mathrm{~g}$ egg at $25^{\circ} \mathrm{C}$ under spray drier factors inlet air temperature $\left(180^{\circ} \mathrm{C}\right)$, feed flow rate $(300 \mathrm{~mL} / \mathrm{hr})$, atomization speed $(24,000 \mathrm{rpm})$ and outlet

Table 5 Impact of Spray drying conditions on eicosapentaenoic fatty acids retention in designer egg dried powder at different days and storage intervals

\begin{tabular}{|c|c|c|c|c|c|c|c|c|c|}
\hline \multirow{3}{*}{$\begin{array}{l}\text { Spray } \\
\text { dryer } \\
\text { process } \\
\text { run }\end{array}$} & \multicolumn{4}{|c|}{ Independent variables } & \multicolumn{5}{|c|}{ EPA (mg/50 g egg) } \\
\hline & \multirow{2}{*}{$\begin{array}{l}\text { Inlet air } \\
\text { temperature } \\
\left({ }^{\circ} \mathrm{C}\right)\end{array}$} & \multirow{2}{*}{$\begin{array}{l}\text { Feed } \\
\text { flow } \\
\text { rate } \\
(\mathrm{mL} / \mathrm{hr})\end{array}$} & \multirow{2}{*}{$\begin{array}{l}\text { Atomization } \\
\text { speed (rpm) }\end{array}$} & \multirow{2}{*}{$\begin{array}{l}\text { Outlet } \\
\text { temperature } \\
\left({ }^{\circ} \mathrm{C}\right)\end{array}$} & \multirow[t]{2}{*}{ o Day } & \multicolumn{2}{|c|}{ Storage at Temperature $4^{\circ} \mathrm{C}$} & \multicolumn{2}{|c|}{ Storage at Temperature $25^{\circ} \mathrm{C}$} \\
\hline & & & & & & 30 Days & 60 Days & 30 Days & 60 Days \\
\hline 1 & $160(-1)$ & $300(0)$ & $16,000(-1)$ & $70(0)$ & $14.51 \pm 0.46^{a}$ & $13.22 \pm 0.41^{\mathrm{ab}}$ & $9.76 \pm 0.41^{c d}$ & $10.92 \pm 0.41^{c}$ & $5.14 \pm 0.44^{\mathrm{ef}}$ \\
\hline 2 & $180(0)$ & $200(-1)$ & $20,000(0)$ & $60(-1)$ & $13.55 \pm 0.38^{\mathrm{ab}}$ & $12.15 \pm 0.35^{b}$ & $8.81 \pm 0.35^{d}$ & $9.91 \pm 0.35^{\mathrm{cd}}$ & $4.75 \pm 0.32^{f}$ \\
\hline 3 & $180(0)$ & $300(0)$ & $16,000(-1)$ & $80(+1)$ & $13.22 \pm 0.34^{\mathrm{ab}}$ & $12.11 \pm 0.32^{b}$ & $8.40 \pm 0.32^{d}$ & $9.84 \pm 0.32^{\mathrm{cd}}$ & $4.72 \pm 0.31^{f}$ \\
\hline 4 & $180(0)$ & $400(+1)$ & $20,000(0)$ & $60(-1)$ & $14.25 \pm 0.41^{\mathrm{a}}$ & $13.05 \pm 0.40^{\mathrm{ab}}$ & $9.42 \pm 0.40^{c d}$ & $10.89 \pm 0.40^{c}$ & $5.69 \pm 0.34^{\mathrm{ef}}$ \\
\hline 5 & $180(0)$ & $200(-1)$ & $16,000(-1)$ & $70(0)$ & $13.45 \pm 0.39^{\mathrm{ab}}$ & $12.19 \pm 0.35^{b}$ & $5.58 \pm 0.35^{\mathrm{ef}}$ & $9.86 \pm 0.35^{\mathrm{cd}}$ & $4.88 \pm 0.32^{f}$ \\
\hline 6 & $160(-1)$ & $300(0)$ & $24,000(+1)$ & $70(0)$ & $13.05 \pm 0.33^{\mathrm{ab}}$ & $12.00 \pm 0.31^{b}$ & $8.82 \pm 0.31^{d}$ & $9.00 \pm 0.31^{c d}$ & $4.75 \pm 0.29^{f}$ \\
\hline $7\left(C_{1}\right)$ & $180(0)$ & $300(0)$ & $20,000(0)$ & $70(0)$ & $13.09 \pm 0.34^{\mathrm{ab}}$ & $12.03 \pm 0.32^{b}$ & $8.86 \pm 0.32^{d}$ & $9.74 \pm 0.32^{\mathrm{cd}}$ & $4.65 \pm 0.30^{f}$ \\
\hline $8\left(C_{2}\right)$ & $180(0)$ & $300(0)$ & $20,000(0)$ & $70(0)$ & $13.10 \pm 0.38^{\mathrm{ab}}$ & $12.15 \pm 0.37^{b}$ & $8.87 \pm 0.37^{d}$ & $9.72 \pm 0.37^{c d}$ & $4.61 \pm 0.31^{f}$ \\
\hline 9 & $180(0)$ & $300(0)$ & $24,000(+1)$ & $60(-1)$ & $13.05 \pm 0.39^{\mathrm{ab}}$ & $12.11 \pm 0.35^{b}$ & $8.83 \pm 0.35^{d}$ & $9.82 \pm 0.35^{c d}$ & $4.67 \pm 0.38^{f}$ \\
\hline 10 & $200(+1)$ & $300(0)$ & $24,000(+1)$ & $70(0)$ & $11.53 \pm 0.29^{b c}$ & $10.05 \pm 0.21^{c}$ & $6.90 \pm 0.21^{e}$ & $7.99 \pm 0.21^{\text {de }}$ & $2.91 \pm 0.27^{9}$ \\
\hline $11\left(C_{3}\right)$ & $180(0)$ & $300(0)$ & $20,000(0)$ & $70(0)$ & $13.11 \pm 0.33^{\mathrm{ab}}$ & $12.18 \pm 0.30^{b}$ & $8.81 \pm 0.30^{d}$ & $9.91 \pm 0.30^{c d}$ & $4.62 \pm 0.29^{f}$ \\
\hline $12\left(C_{4}\right)$ & $180(0)$ & $300(0)$ & $20,000(0)$ & $70(0)$ & $13.12 \pm 0.38^{\mathrm{ab}}$ & $12.11 \pm 0.33^{b}$ & $8.82 \pm 0.33^{d}$ & $9.92 \pm 0.33^{\mathrm{cd}}$ & $4.63 \pm 0.31^{f}$ \\
\hline 13 & $180(0)$ & $200(-1)$ & $20,000(0)$ & $80(+1)$ & $12.15 \pm 0.28^{b}$ & $11.09 \pm 0.22^{b c}$ & $7.11 \pm 0.22^{\text {de }}$ & $8.00 \pm 0.22^{d}$ & $3.89 \pm 0.18^{f g}$ \\
\hline 14 & $200(+1)$ & $400(+1)$ & $20,000(0)$ & $70(0)$ & $12.72 \pm 0.26^{b}$ & $11.18 \pm 0.20^{b c}$ & $7.71 \pm 0.20^{\mathrm{de}}$ & $8.82 \pm 0.20^{d}$ & $3.35 \pm 0.23^{f g}$ \\
\hline 15 & $160(-1)$ & $400(+1)$ & $20,000(0)$ & $70(0)$ & $14.25 \pm 0.45^{\mathrm{a}}$ & $13.03 \pm 0.41^{\mathrm{ab}}$ & $9.15 \pm 0.41^{c d}$ & $10.45 \pm 0.41^{c}$ & $5.88 \pm 0.28^{\mathrm{ef}}$ \\
\hline 16 & $180(0)$ & $400(+1)$ & $20,000(0)$ & $80(+1)$ & $12.93 \pm 0.29^{b}$ & $11.53 \pm 0.21^{b c}$ & $7.35 \pm 0.21^{\mathrm{de}}$ & $8.83 \pm 0.21^{d}$ & $3.37 \pm 0.32^{\mathrm{fg}}$ \\
\hline 17 & $160(-1)$ & $300(0)$ & $20,000(0)$ & $80(+1)$ & $13.24 \pm 0.35^{\mathrm{ab}}$ & $12.06 \pm 0.33^{b}$ & $8.34 \pm 0.33^{d}$ & $9.91 \pm 0.33^{c d}$ & $4.67 \pm 0.39^{f}$ \\
\hline 18 & $200(+1)$ & $300(0)$ & $20,000(0)$ & $80(+1)$ & $11.75 \pm 0.27^{\mathrm{bc}}$ & $10.02 \pm 0.21^{c}$ & $6.98 \pm 0.21^{e}$ & $7.11 \pm 0.21^{\mathrm{de}}$ & $2.29 \pm 0.15^{9}$ \\
\hline 19 & $160(-1)$ & $200(-1)$ & $20,000(0)$ & $70(0)$ & $13.45 \pm 0.39^{\mathrm{ab}}$ & $12.19 \pm 0.31^{b}$ & $8.31 \pm 0.31^{d}$ & $9.91 \pm 0.31^{\mathrm{cd}}$ & $4.22 \pm 0.21^{f}$ \\
\hline 20 & $180(0)$ & $400(+1)$ & $16,000(-1)$ & $70(0)$ & $14.26 \pm 0.42^{a}$ & $13.10 \pm 0.40^{\mathrm{ab}}$ & $9.34 \pm 0.40^{c d}$ & $10.13 \pm 0.40^{c}$ & $5.67 \pm 0.23^{\mathrm{ef}}$ \\
\hline 21 & $180(0)$ & $300(0)$ & $24,000(+1)$ & $80(+1)$ & $11.78 \pm 0.31^{b c}$ & $10.35 \pm 0.28^{c}$ & $6.70 \pm 0.28^{e}$ & $7.00 \pm 0.28^{\mathrm{de}}$ & $2.18 \pm 0.14^{g}$ \\
\hline 22 & $180(0)$ & $300(0)$ & $16,000(-1)$ & $60(-1)$ & $14.55 \pm 0.46^{\mathrm{a}}$ & $13.10 \pm 0.37^{\mathrm{ab}}$ & $9.59 \pm 0.37^{c d}$ & $10.33 \pm 0.37^{c}$ & $5.91 \pm 0.27^{e f}$ \\
\hline 23 & $180(0)$ & $400(+1)$ & $24,000(+1)$ & $70(0)$ & $12.72 \pm 0.34^{b}$ & $11.31 \pm 0.32^{\mathrm{bc}}$ & $7.93 \pm 0.32^{\mathrm{de}}$ & $8.82 \pm 0.32^{d}$ & $3.89 \pm 0.30^{f g}$ \\
\hline 24 & $160(-1)$ & $300(0)$ & $20,000(0)$ & $60(-1)$ & $14.55 \pm 0.41^{\mathrm{a}}$ & $13.13 \pm 0.38^{\mathrm{ab}}$ & $9.83 \pm 0.38^{\mathrm{cd}}$ & $10.81 \pm 0.38^{c}$ & $5.86 \pm 0.25^{\mathrm{ef}}$ \\
\hline $25\left(C_{5}\right)$ & $180(0)$ & $300(0)$ & $20,000(0)$ & $70(0)$ & $13.08 \pm 0.33^{\mathrm{ab}}$ & $12.14 \pm 0.30^{b}$ & $8.85 \pm 0.30^{d}$ & $9.71 \pm 0.30^{\mathrm{cd}}$ & $4.51 \pm 0.21^{f}$ \\
\hline 26 & $200(+1)$ & $300(0)$ & $16,000(-1)$ & $70(0)$ & $13.05 \pm 0.37^{\mathrm{ab}}$ & $12.31 \pm 0.31^{b}$ & $8.01 \pm 0.31^{d}$ & $9.09 \pm 0.31^{\mathrm{cd}}$ & $4.42 \pm 0.28^{f}$ \\
\hline 27 & $180(0)$ & $200(-1)$ & $24,000(+1)$ & $70(0)$ & $11.95 \pm 0.29^{b c}$ & $10.72 \pm 0.22^{c}$ & $6.02 \pm 0.22^{e}$ & $7.22 \pm 0.22^{\mathrm{de}}$ & $2.39 \pm 0.11^{9}$ \\
\hline 28 & $200(+1)$ & $200(-1)$ & $20,000(0)$ & $70(0)$ & $11.94 \pm 0.32^{b c}$ & $10.70 \pm 0.29^{c}$ & $6.00 \pm 0.29^{e}$ & $7.73 \pm 0.29^{\text {de }}$ & $2.26 \pm 0.19^{9}$ \\
\hline 29 & $200(+1)$ & $300(0)$ & $20,000(0)$ & $60(-1)$ & $13.05 \pm 0.36^{\mathrm{ab}}$ & $12.10 \pm 0.31^{b}$ & $8.11 \pm 0.31^{d}$ & $9.71 \pm 0.31^{c d}$ & $4.54 \pm 0.36^{f}$ \\
\hline
\end{tabular}

$\mathrm{C} 1, \mathrm{C} 2, \mathrm{C} 3, \mathrm{C} 4, \mathrm{C} 5$ represent spraying drying process at center points

Experimental model $=$ Box-Behnken design

Total number of spray drying treatments $=29$

No of replicates $=03$

${ }^{\mathrm{a}-\mathrm{g}}$ values with similar letters show homogenous group within row and column $(p>0.05)$ 
air temperature $\left(80^{\circ} \mathrm{C}\right)$ after 60 days storage period. The EPA changes were $31.13 \%$ (at $4{ }^{\circ} \mathrm{C}$ after 30 days), $61.64 \%$ (at $4{ }^{\circ} \mathrm{C}$ after 60 days), $51.89 \%$ (at $25^{\circ} \mathrm{C}$ after 30 days) and $85.01 \%$ (at $25^{\circ} \mathrm{C}$ after 60 days), respectively.

The minimum values for DEDP samples regarding to retention of docosahexaenoic fatty acids stored at two different storage temperatures $4{ }^{\circ} \mathrm{C}$ and $25^{\circ} \mathrm{C}$ observed at the same spray drier conditions ( 0,30 and 60 days $)$ as shown in Table 6. The DHA value in DEDP was decreased from $15.49 \pm 0.79 \mathrm{mg} / 50 \mathrm{~g}$ egg ( 0 day) to 10.10 $\pm 0.64 \mathrm{mg} / 50 \mathrm{~g}$ egg at 60 days $\left(4^{\circ} \mathrm{C}\right)$ and same decreasing trend was observed at $25^{\circ} \mathrm{C}$. The trend in percent changes calculated for EPA was $8.26 \%$ (at $4{ }^{\circ} \mathrm{C}$ after 30 days), $34.79 \%$ (at $4{ }^{\circ} \mathrm{C}$ after 60 days), $27.88 \%$ (at $25^{\circ} \mathrm{C}$ after 30 days) and $61.20 \%$ (at $25^{\circ} \mathrm{C}$ after 60 days), respectively. The decreasing order for total omega-3 fatty acids retention in DEDP obtained by keeping spray drier factors (i.e. inlet air temperature and feed flow rate at

Table 6 Impact of Spray drying conditions on docosahexaenoic fatty acids retention in designer egg dried powder at different days and storage intervals

\begin{tabular}{|c|c|c|c|c|c|c|c|c|c|}
\hline \multirow{3}{*}{$\begin{array}{l}\text { Spray } \\
\text { dryer } \\
\text { process } \\
\text { run }\end{array}$} & \multicolumn{4}{|c|}{ Independent variables } & \multicolumn{5}{|c|}{$\mathrm{DHA}(\mathrm{mg} / 50 \mathrm{~g}$ egg) } \\
\hline & \multirow{2}{*}{$\begin{array}{l}\text { Inlet air } \\
\text { temperature } \\
\left({ }^{\circ} \mathrm{C}\right)\end{array}$} & \multirow{2}{*}{$\begin{array}{l}\text { Feed } \\
\text { flow } \\
\text { rate } \\
(\mathrm{mL} / \mathrm{hr})\end{array}$} & \multirow{2}{*}{$\begin{array}{l}\text { Atomization } \\
\text { speed (rpm) }\end{array}$} & \multirow{2}{*}{$\begin{array}{l}\text { Outlet } \\
\text { temperature } \\
\left({ }^{\circ} \mathrm{C}\right)\end{array}$} & \multirow[t]{2}{*}{ o Day } & \multicolumn{2}{|c|}{ Storage at Temperature $4^{\circ} \mathrm{C}$} & \multicolumn{2}{|c|}{ Storage at Temperature $25^{\circ} \mathrm{C}$} \\
\hline & & & & & & 30 Days & 60 Days & 30 Days & 60 Days \\
\hline 1 & $160(-1)$ & $300(0)$ & $16,000(-1)$ & $70(0)$ & $19.46 \pm 0.88^{a}$ & $18.21 \pm 0.81^{\mathrm{ab}}$ & $14.15 \pm 0.79^{\text {cd }}$ & $15.15 \pm 0.79^{c}$ & $10.45 \pm 0.73^{e f}$ \\
\hline 2 & $180(0)$ & $200(-1)$ & $20,000(0)$ & $60(-1)$ & $18.07 \pm 0.85^{\mathrm{ab}}$ & $17.85 \pm 0.71^{b}$ & $13.65 \pm 0.70^{d}$ & $14.65 \pm 0.70^{c d}$ & $9.63 \pm 0.71^{f}$ \\
\hline 3 & $180(0)$ & $300(0)$ & $16,000(-1)$ & $80(+1)$ & $17.62 \pm 0.79^{b}$ & $16.40 \pm 0.74^{b c}$ & $12.35 \pm 0.71^{\text {de }}$ & $13.55 \pm 0.71^{d}$ & $8.55 \pm 0.67^{\mathrm{fg}}$ \\
\hline 4 & $180(0)$ & $400(+1)$ & $20,000(0)$ & $60(-1)$ & $19.32 \pm 0.72^{a}$ & $18.20 \pm 0.69^{a b}$ & $14.11 \pm 0.63^{\mathrm{cd}}$ & $15.22 \pm 0.63^{c}$ & $10.76 \pm 0.64^{\mathrm{ef}}$ \\
\hline 5 & $180(0)$ & $200(-1)$ & $16,000(-1)$ & $70(0)$ & $18.45 \pm 0.73^{\mathrm{ab}}$ & $17.21 \pm 0.68^{b}$ & $13.18 \pm 0.61^{d}$ & $14.00 \pm 0.61^{c d}$ & $9.67 \pm 0.65^{f}$ \\
\hline 6 & $160(-1)$ & $300(0)$ & $24,000(+1)$ & $70(0)$ & $17.42 \pm 0.81^{b}$ & $16.12 \pm 0.78^{b c}$ & $12.02 \pm 0.75^{\text {de }}$ & $13.51 \pm 0.75^{d}$ & $8.45 \pm 0.71^{\mathrm{fg}}$ \\
\hline $7\left(C_{1}\right)$ & $180(0)$ & $300(0)$ & $20,000(0)$ & $70(0)$ & $17.44 \pm 0.80^{b}$ & $16.11 \pm 0.71^{b c}$ & $12.01 \pm 0.70^{\text {de }}$ & $13.59 \pm 0.70^{d}$ & $8.41 \pm 0.63^{\mathrm{fg}}$ \\
\hline $8\left(C_{2}\right)$ & $180(0)$ & $300(0)$ & $20,000(0)$ & $70(0)$ & $17.46 \pm 0.78^{b}$ & $16.13 \pm 0.72^{b c}$ & $12.03 \pm 0.71^{\text {de }}$ & $13.58 \pm 0.71^{d}$ & $8.66 \pm 0.71^{\mathrm{fg}}$ \\
\hline 9 & $180(0)$ & $300(0)$ & $24,000(+1)$ & $60(-1)$ & $17.48 \pm 0.82^{b}$ & $16.18 \pm 0.74^{b c}$ & $12.09 \pm 0.75^{\mathrm{de}}$ & $13.52 \pm 0.75^{d}$ & $8.72 \pm 0.72^{f g}$ \\
\hline 10 & $200(+1)$ & $300(0)$ & $24,000(+1)$ & $70(0)$ & $15.49 \pm 0.79^{c}$ & $14.21 \pm 0.69^{c d}$ & $10.10 \pm 0.64^{e f}$ & $11.17 \pm 0.64^{e}$ & $6.01 \pm 0.63^{h}$ \\
\hline $11\left(C_{3}\right)$ & $180(0)$ & $300(0)$ & $20,000(0)$ & $70(0)$ & $17.41 \pm 0.83^{b}$ & $16.15 \pm 0.81^{b c}$ & $12.04 \pm 0.88^{\mathrm{de}}$ & $13.49 \pm 0.88^{d}$ & $8.58 \pm 0.81^{\mathrm{fg}}$ \\
\hline $12\left(C_{4}\right)$ & $180(0)$ & $300(0)$ & $20,000(0)$ & $70(0)$ & $17.43 \pm 0.77^{b}$ & $16.19 \pm 0.70^{b c}$ & $12.11 \pm 0.73^{\text {de }}$ & $13.33 \pm 0.73^{d}$ & $8.49 \pm 0.73^{\mathrm{fg}}$ \\
\hline 13 & $180(0)$ & $200(-1)$ & $20,000(0)$ & $80(+1)$ & $16.27 \pm 0.84^{b c}$ & $15.00 \pm 0.72^{c}$ & $11.85 \pm 0.81^{e}$ & $12.89 \pm 0.81^{\mathrm{de}}$ & $7.54 \pm 0.82^{9}$ \\
\hline 14 & $200(+1)$ & $400(+1)$ & $20,000(0)$ & $70(0)$ & $17.08 \pm 0.76^{b}$ & $16.22 \pm 0.71^{b c}$ & $12.66 \pm 0.73^{\mathrm{de}}$ & $13.77 \pm 0.73^{d}$ & $8.09 \pm 0.71^{\mathrm{fg}}$ \\
\hline 15 & $160(-1)$ & $400(+1)$ & $20,000(0)$ & $70(0)$ & $19.11 \pm 0.72^{a}$ & $18.35 \pm 0.70^{\mathrm{ab}}$ & $14.63 \pm 0.71^{c d}$ & $15.79 \pm 0.71^{\mathrm{C}}$ & $10.00 \pm 0.72^{e f}$ \\
\hline 16 & $180(0)$ & $400(+1)$ & $20,000(0)$ & $80(+1)$ & $17.23 \pm 0.85^{b}$ & $16.01 \pm 0.88^{b c}$ & $12.89 \pm 0.83^{\mathrm{de}}$ & $13.91 \pm 0.83^{d}$ & $8.55 \pm 0.83^{\mathrm{fg}}$ \\
\hline 17 & $160(-1)$ & $300(0)$ & $20,000(0)$ & $80(+1)$ & $17.65 \pm 0.78^{b}$ & $16.39 \pm 0.72^{b c}$ & $12.21 \pm 0.76^{\text {de }}$ & $13.41 \pm 0.76^{d}$ & $8.68 \pm 0.75^{\mathrm{fg}}$ \\
\hline 18 & $200(+1)$ & $300(0)$ & $20,000(0)$ & $80(+1)$ & $15.62 \pm 0.86^{c}$ & $14.39 \pm 0.82^{c d}$ & $10.23 \pm 0.85^{e f}$ & $11.81 \pm 0.85^{e}$ & $6.58 \pm 0.84^{h}$ \\
\hline 19 & $160(-1)$ & $200(-1)$ & $20,000(0)$ & $70(0)$ & $18.10 \pm 0.89^{a b}$ & $17.90 \pm 0.83^{b}$ & $13.59 \pm 0.84^{d}$ & $14.73 \pm 0.84^{\text {cd }}$ & $9.55 \pm 0.82^{f}$ \\
\hline 20 & $180(0)$ & $400(+1)$ & $16,000(-1)$ & $70(0)$ & $19.03 \pm 0.91^{a}$ & $18.79 \pm 0.93^{a b}$ & $14.61 \pm 0.92^{c d}$ & $15.69 \pm 0.92^{c}$ & $10.80 \pm 0.91^{\mathrm{ef}}$ \\
\hline 21 & $180(0)$ & $300(0)$ & $24,000(+1)$ & $80(+1)$ & $15.62 \pm 0.69^{c}$ & $14.33 \pm 0.63^{c d}$ & $10.11 \pm 0.65^{e f}$ & $11.21 \pm 0.65^{e}$ & $6.78 \pm 0.65^{h}$ \\
\hline 22 & $180(0)$ & $300(0)$ & $16,000(-1)$ & $60(-1)$ & $19.41 \pm 0.92^{\mathrm{a}}$ & $18.19 \pm 0.96^{\mathrm{ab}}$ & $14.01 \pm 0.93^{c d}$ & $15.31 \pm 0.93^{c}$ & $10.11 \pm 0.89^{e f}$ \\
\hline 23 & $180(0)$ & $400(+1)$ & $24,000(+1)$ & $70(0)$ & $17.02 \pm 0.75^{b}$ & $16.60 \pm 0.71^{b c}$ & $12.49 \pm 0.72^{\text {de }}$ & $13.69 \pm 0.72^{d}$ & $8.41 \pm 0.74^{\mathrm{fg}}$ \\
\hline 24 & $160(-1)$ & $300(0)$ & $20,000(0)$ & $60(-1)$ & $19.43 \pm 0.88^{a}$ & $18.40 \pm 0.82^{\mathrm{ab}}$ & $14.23 \pm 0.85^{c d}$ & $15.99 \pm 0.85^{c}$ & $10.21 \pm 0.85^{e f}$ \\
\hline $25\left(C_{5}\right)$ & $180(0)$ & $300(0)$ & $20,000(0)$ & $70(0)$ & $17.40 \pm 0.72^{b}$ & $16.22 \pm 0.76^{b c}$ & $12.09 \pm 0.79^{\text {de }}$ & $13.40 \pm 0.79^{d}$ & $8.55 \pm 0.75^{\mathrm{fg}}$ \\
\hline 26 & $200(+1)$ & $300(0)$ & $16,000(-1)$ & $70(0)$ & $17.45 \pm 0.82^{b}$ & $16.25 \pm 0.79^{b c}$ & $12.10 \pm 0.80^{\text {de }}$ & $13.05 \pm 0.80^{d}$ & $8.59 \pm 0.84^{f g}$ \\
\hline 27 & $180(0)$ & $200(-1)$ & $24,000(+1)$ & $70(0)$ & $16.04 \pm 0.77^{b c}$ & $15.30 \pm 0.71^{c}$ & $11.63 \pm 0.75^{e}$ & $12.91 \pm 0.75^{\text {de }}$ & $7.98 \pm 0.73^{9}$ \\
\hline 28 & $200(+1)$ & $200(-1)$ & $20,000(0)$ & $70(0)$ & $16.09 \pm 0.79^{b c}$ & $15.30 \pm 0.74^{c}$ & $11.51 \pm 0.76^{e}$ & $12.72 \pm 0.76^{\text {de }}$ & $7.94 \pm 0.72^{9}$ \\
\hline 29 & $200(+1)$ & $300(0)$ & $20,000(0)$ & $60(-1)$ & $17.46 \pm 0.83^{b}$ & $16.22 \pm 0.81^{b c}$ & $12.00 \pm 0.82^{\mathrm{de}}$ & $13.20 \pm 0.82^{d}$ & $8.12 \pm 0.82^{f g}$ \\
\hline
\end{tabular}

$\mathrm{C1}, \mathrm{C2}, \mathrm{C3}, \mathrm{C4}, \mathrm{C5}$ represent spraying drying process at center points

Experimental model $=$ Box-Behnken design

Total number of spray drying treatments $=29$

No of replicates $=03$

${ }^{a-h}$ values with similar letters show homogenous group within row and column $(p>0.05)$ 
medium level whereas atomization and outlet air temperature at minimum level) during storage intervals was found $162.33 \pm 1.64 \mathrm{mg} / 50 \mathrm{~g}$ egg $>158.61 \pm 1.53 \mathrm{mg} /$ $50 \mathrm{~g}$ egg $>148.03 \pm 1.57 \mathrm{mg} / 50 \mathrm{~g}$ egg ( 0,30 and 60 days stored at $\left.4{ }^{\circ} \mathrm{C}\right)$ and $162.33 \pm 1.64 \mathrm{mg} / 50 \mathrm{~g}$ egg $>151.56 \pm$ $1.54 \mathrm{mg} / 50 \mathrm{~g}$ egg $>135.89 \pm 1.62 \mathrm{mg} / 50 \mathrm{~g}$ egg $(0,30$ and 60 days stored at $25^{\circ} \mathrm{C}$ ) (Table 7 ).

The effects of various spray drier conditions and storage on peroxide value in DEDP have been shown in Table 8. The PV of DEDP samples reached their maximum peaks after 60 days at $25^{\circ} \mathrm{C}$. The increasing order shows that lipid oxidation increased with storage. The peroxides are considered as early oxidation products with relatively short induction periods during which they form, accumulate and dissipate. It seems true that the DEDP samples stored for 30 days at lower temperature were relatively stable than stored at higher temperature for 60 days. The overall PV never exceeded the limit of $10(\mathrm{meq} / \mathrm{kg})$ considered as a threshold limit. The PV levels obtained from 60 days in DEDP

Table 7 Impact of Spray drying conditions on total omega-3 fatty acids retention in designer egg dried powder at different days and storage intervals

\begin{tabular}{|c|c|c|c|c|c|c|c|c|c|}
\hline \multirow{3}{*}{$\begin{array}{l}\text { Spray } \\
\text { dryer } \\
\text { process } \\
\text { run }\end{array}$} & \multicolumn{4}{|c|}{ Independent variables } & \multicolumn{5}{|c|}{ Total omega-3 fatty acids (mg/50 g egg) } \\
\hline & \multirow{2}{*}{$\begin{array}{l}\text { Inlet air } \\
\text { temperature } \\
\left({ }^{\circ} \mathrm{C}\right)\end{array}$} & \multirow{2}{*}{$\begin{array}{l}\text { Feed flow } \\
\text { rate }(\mathrm{mL} / \mathrm{hr})\end{array}$} & \multirow{2}{*}{$\begin{array}{l}\text { Atomization } \\
\text { speed (rpm) }\end{array}$} & \multirow{2}{*}{$\begin{array}{l}\text { Outlet } \\
\text { temperature } \\
\left({ }^{\circ} \mathrm{C}\right)\end{array}$} & \multirow[t]{2}{*}{ ODay } & \multicolumn{2}{|c|}{ Storage at Temperature $4^{\circ} \mathrm{C}$} & \multicolumn{2}{|c|}{ Storage at Temperature $25^{\circ} \mathrm{C}$} \\
\hline & & & & & & 30 Days & 60 Days & 30 Days & 60 Days \\
\hline 1 & $160(-1)$ & $300(0)$ & $16,000(-1)$ & $70(0)$ & $161.54 \pm 2.15^{\mathrm{ab}}$ & $157.65 \pm 2.21^{c}$ & $146.96 \pm 2.28^{g h}$ & $149.19 \pm 2.14^{f}$ & $134.11 \pm 2.11^{\mathrm{m}}$ \\
\hline 2 & $180(0)$ & $200(-1)$ & $20,000(0)$ & $60(-1)$ & $150.43 \pm 2.14^{\mathrm{ef}}$ & $147.34 \pm 2.22^{9}$ & $136.79 \pm 2.07^{1}$ & $138.81 \pm 2.01^{\mathrm{k}}$ & $123.93 \pm 2.02^{r}$ \\
\hline 3 & $180(0)$ & $300(0)$ & $16,000(-1)$ & $80(+1)$ & $147.40 \pm 2.47^{9}$ & $145.7 \pm 2.33^{h}$ & $134.89 \pm 2.12^{\mathrm{m}}$ & $135.76 \pm 1.80^{\mathrm{Im}}$ & $120.08 \pm 1.93 \mathrm{st}$ \\
\hline 4 & $180(0)$ & $400(+1)$ & $20,000(0)$ & $60(-1)$ & $159.34 \pm 1.88^{b}$ & $155.3 \pm 1.84^{d}$ & $145.29 \pm 1.91^{h}$ & $147.64 \pm 1.65^{9}$ & $132.88 \pm 1.44^{n}$ \\
\hline 5 & $180(0)$ & $200(-1)$ & $16,000(-1)$ & $70(0)$ & $150.20 \pm 2.11^{\mathrm{ef}}$ & $146.44 \pm 1.94^{g h}$ & $133.51 \pm 1.72^{\mathrm{mn}}$ & $138.07 \pm 1.76^{k}$ & $124.06 \pm 1.95^{\mathrm{qr}}$ \\
\hline 6 & $160(-1)$ & $300(0)$ & $24,000(+1)$ & $70(0)$ & $145.22 \pm 1.88^{h}$ & $141.78 \pm 1.53^{\mathrm{ij}}$ & $131.29 \pm 1.61^{\mathrm{no}}$ & $133.06 \pm 1.57^{\mathrm{mn}}$ & $119.07 \pm 1.44^{t}$ \\
\hline $7\left(C_{1}\right)$ & $180(0)$ & $300(0)$ & $20,000(0)$ & $70(0)$ & $145.35 \pm 1.52^{h}$ & $141.69 \pm 1.88^{\mathrm{ij}}$ & $131.63 \pm 1.85^{\mathrm{no}}$ & $134.75 \pm 1.82^{\mathrm{m}}$ & $118.77 \pm 1.98^{\mathrm{tu}}$ \\
\hline $8\left(C_{2}\right)$ & $180(0)$ & $300(0)$ & $20,000(0)$ & $70(0)$ & $145.24 \pm 1.57^{h}$ & $141.52 \pm 1.85^{\mathrm{ij}}$ & $131.31 \pm 1.96^{\mathrm{no}}$ & $134.72 \pm 1.74^{\mathrm{m}}$ & $118.85 \pm 1.96^{\text {tu }}$ \\
\hline 9 & $180(0)$ & $300(0)$ & $24,000(+1)$ & $60(-1)$ & $146.5 \pm 1.78^{g h}$ & $142.91 \pm 1.84^{i}$ & $132.87 \pm 1.81^{n}$ & $134.16 \pm 1.65^{\mathrm{m}}$ & $119.84 \pm 1.74^{t}$ \\
\hline 10 & $200(+1)$ & $300(0)$ & $24,000(+1)$ & $70(0)$ & $128.21 \pm 1.55^{\mathrm{p}}$ & $124.41 \pm 1.15^{\mathrm{qr}}$ & $114.16 \pm 1.32^{\mathrm{vw}}$ & $116.71 \pm 1.46^{\mathrm{uv}}$ & $101.60 \pm 1.65^{y}$ \\
\hline $11\left(C_{3}\right)$ & $180(0)$ & $300(0)$ & $20,000(0)$ & $70(0)$ & $145.28 \pm 1.69^{h}$ & $141.78 \pm 1.58^{\mathrm{ij}}$ & $131.46 \pm 1.75^{\mathrm{no}}$ & $134.81 \pm 1.84^{\mathrm{m}}$ & $118.63 \pm 1.78^{\text {tu }}$ \\
\hline $12\left(C_{4}\right)$ & $180(0)$ & $300(0)$ & $20,000(0)$ & $70(0)$ & $145.30 \pm 1.63^{h}$ & $141.81 \pm 1.52^{i j}$ & $131.56 \pm 1.89^{\mathrm{no}}$ & $134.68 \pm 1.62^{\mathrm{m}}$ & $118.78 \pm 1.68^{\mathrm{tu}}$ \\
\hline 13 & $180(0)$ & $200(-1)$ & $20,000(0)$ & $80(+1)$ & $134.38 \pm 1.81^{\mathrm{m}}$ & $131.53 \pm 1.61^{\text {no }}$ & $121.51 \pm 1.72^{5}$ & $123.1 \pm 1.74^{r}$ & $108.99 \pm 1.83^{w}$ \\
\hline 14 & $200(+1)$ & $400(+1)$ & $20,000(0)$ & $70(0)$ & $140.97 \pm 1.95^{j}$ & $137.45 \pm 1.84^{k l}$ & $127.98 \pm 1.81^{p q}$ & $129.9 \pm 1.96^{\circ p}$ & $114.05 \pm 1.75^{\mathrm{vw}}$ \\
\hline 15 & $160(-1)$ & $400(+1)$ & $20,000(0)$ & $70(0)$ & $157.92 \pm 1.74^{c}$ & $154.93 \pm 1.65^{\mathrm{de}}$ & $144.53 \pm 1.62^{h i}$ & $146.86 \pm 1.56^{\mathrm{gh}}$ & $131.33 \pm 1.55^{\text {no }}$ \\
\hline 16 & $180(0)$ & $400(+1)$ & $20,000(0)$ & $80(+1)$ & $154.08 \pm 1.61^{\mathrm{de}}$ & $140.19 \pm 1.57^{j}$ & $129.98 \pm 1.54^{\mathrm{op}}$ & $132.06 \pm 1.56^{n}$ & $116.25 \pm 1.57^{\mathrm{uv}}$ \\
\hline 17 & $160(-1)$ & $300(0)$ & $20,000(0)$ & $80(+1)$ & $146.42 \pm 1.84^{g h}$ & $142.57 \pm 1.75^{i}$ & $131.85 \pm 1.87^{\mathrm{no}}$ & $134.78 \pm 1.71^{\mathrm{m}}$ & $120.13 \pm 1.76 \mathrm{st}$ \\
\hline 18 & $200(+1)$ & $300(0)$ & $20,000(0)$ & $80(+1)$ & $130.0 \pm 1.34^{\circ}$ & $126.03 \pm 1.23^{9}$ & $115.54 \pm 1.26^{v}$ & $117.55 \pm 1.21^{\mathrm{u}}$ & $102.85 \pm 1.32^{x y}$ \\
\hline 19 & $160(-1)$ & $200(-1)$ & $20,000(0)$ & $70(0)$ & $149.42 \pm 1.55^{f}$ & $146.2 \pm 1.44^{g h}$ & $135.49 \pm 1.48^{\mathrm{lm}}$ & $137.79 \pm 1.58^{\mathrm{kl}}$ & $121.86 \pm 1.41^{\mathrm{s}}$ \\
\hline 20 & $180(0)$ & $400(+1)$ & $16,000(-1)$ & $70(0)$ & $158.93 \pm 1.72^{\mathrm{bc}}$ & $156.51 \pm 1.61^{\mathrm{cd}}$ & $145.27 \pm 1.85^{h}$ & $147.25 \pm 1.74^{9}$ & $132.82 \pm 1.68^{n}$ \\
\hline 21 & $180(0)$ & $300(0)$ & $24,000(+1)$ & $80(+1)$ & $131.24 \pm 1.91^{\mathrm{no}}$ & $126.69 \pm 1.82^{9}$ & $116.54 \pm 1.64^{\mathrm{uv}}$ & $117.8 \pm 1.88^{u}$ & $103.63 \pm 1.77^{x}$ \\
\hline 22 & $180(0)$ & $300(0)$ & $000(-1)$ & $60(-1)$ & $162.33 \pm 1.64^{\mathrm{a}}$ & $158.61 \pm 1.53^{b c}$ & $148.03 \pm 1.57^{\mathrm{fg}}$ & $151.56 \pm 1.54^{\mathrm{e}}$ & $135.89 \pm 1.62^{\mathrm{lm}}$ \\
\hline 23 & $180(0)$ & $400(+1)$ & $24,000(+1)$ & $70(0)$ & $141.97 \pm 1.46^{\mathrm{ij}}$ & $139.86 \pm 1.45^{j \mathrm{k}}$ & $128.53 \pm 1.46^{\mathrm{P}}$ & $131.54 \pm 1.53^{\mathrm{no}}$ & $115.64 \pm 1.32^{v}$ \\
\hline 24 & $160(-1)$ & $300(0)$ & $20,000(0)$ & $60(-1)$ & $161.45 \pm 1.51^{\mathrm{ab}}$ & $157.86 \pm 1.42^{c}$ & $147.7 \pm 1.55^{9}$ & $150.81 \pm 1.48^{\mathrm{ef}}$ & $134.82 \pm 1.37^{\mathrm{m}}$ \\
\hline $25\left(C_{5}\right)$ & $180(0)$ & $300(0)$ & $20,000(0)$ & $70(0)$ & $145.45 \pm 1.42^{h}$ & $141.67 \pm 1.81^{i j}$ & $131.55 \pm 1.72^{\mathrm{no}}$ & $134.55 \pm 1.93^{\mathrm{m}}$ & $118.95 \pm 1.58^{\text {tu }}$ \\
\hline 26 & $200(+1)$ & $300(0)$ & $16,000(-1)$ & $70(0)$ & $145.38 \pm 1.45^{h}$ & $141.78 \pm 1.44^{\mathrm{ij}}$ & $130.32 \pm 1.33^{\circ}$ & $132.57 \pm 1.32^{n}$ & $118.34 \pm 1.41^{\text {tu }}$ \\
\hline 27 & $180(0)$ & $200(-1)$ & $24,000(+1)$ & $70(0)$ & $133.00 \pm 1.32^{\mathrm{mn}}$ & $130.02 \pm 1.21^{\circ}$ & $118.69 \pm 1.22^{\mathrm{tu}}$ & $122.1 \pm 1.34^{\mathrm{rs}}$ & $106.71 \pm 1.28^{w x}$ \\
\hline 28 & $200(+1)$ & $200(-1)$ & $20,000(0)$ & $70(0)$ & $132.85 \pm 1.25^{n}$ & $129.54 \pm 1.24^{\circ \mathrm{p}}$ & $117.74 \pm 1.28^{\mathrm{u}}$ & $121.01 \pm 1.22^{5}$ & $106.16 \pm 1.31^{w x}$ \\
\hline 29 & $200(+1)$ & $300(0)$ & $20,000(0)$ & $60(-1)$ & $144.7 \pm 1.44^{\mathrm{hi}}$ & $141.44 \pm 1.32^{i j}$ & $130.32 \pm 1.44^{\circ}$ & $133.58 \pm 1.28^{\mathrm{mn}}$ & $117.99 \pm 1.33^{\mathrm{u}}$ \\
\hline
\end{tabular}

$\mathrm{C} 1, \mathrm{C} 2, \mathrm{C}, \mathrm{C4}, \mathrm{C5}$ represent spraying drying process at center points

Experimental model $=$ Box-Behnken design

Total number of spray drying treatments $=29$

No of replicates $=03$

${ }^{a-y}$ values with similar letters show homogenous group within row and column $(p>0.05)$ 
Table 8 Impact of spray drying conditions on peroxide value in designer egg dried powder at different days and storage intervals

\begin{tabular}{|c|c|c|c|c|c|c|c|c|c|}
\hline \multirow{3}{*}{$\begin{array}{l}\text { Spray } \\
\text { dryer } \\
\text { process } \\
\text { run }\end{array}$} & \multicolumn{4}{|c|}{ Independent variables } & \multicolumn{5}{|c|}{ Peroxide value (meq/kg $\mathrm{O}_{2}$ ) } \\
\hline & \multirow{2}{*}{$\begin{array}{l}\text { Inlet air } \\
\text { temperature } \\
\left({ }^{\circ} \mathrm{C}\right)\end{array}$} & \multirow{2}{*}{$\begin{array}{l}\text { Feed } \\
\text { flow } \\
\text { rate } \\
(\mathrm{mL} / \mathrm{hr})\end{array}$} & \multirow{2}{*}{$\begin{array}{l}\text { Atomization } \\
\text { speed (rpm) }\end{array}$} & \multirow{2}{*}{$\begin{array}{l}\text { Outlet } \\
\text { temperature } \\
\left({ }^{\circ} \mathrm{C}\right)\end{array}$} & \multirow[t]{2}{*}{ o Day } & \multicolumn{2}{|c|}{ Storage at Temperature $4^{\circ} \mathrm{C}$} & \multicolumn{2}{|c|}{ Storage at Temperature $25^{\circ} \mathrm{C}$} \\
\hline & & & & & & 30 Days & 60 Days & 30 Days & 60 Days \\
\hline 1 & $160(-1)$ & $300(0)$ & $16,000(-1)$ & $70(0)$ & $0.46 \pm 0.05^{p q}$ & $0.52 \pm 0.01^{n}$ & $0.59 \pm 0.08^{\mathrm{jk}}$ & $0.53 \pm 0.04^{\mathrm{mn}}$ & $0.62 \pm 0.01^{i}$ \\
\hline 2 & $180(0)$ & $200(-1)$ & $20,000(0)$ & $60(-1)$ & $0.45 \pm 0.04^{9}$ & $0.51 \pm 0.02^{\text {no }}$ & $0.58 \pm 0.07^{k}$ & $0.52 \pm 0.01^{n}$ & $0.61 \pm 0.02^{i j}$ \\
\hline 3 & $180(0)$ & $300(0)$ & $16,000(-1)$ & $80(+1)$ & $0.58 \pm 0.07^{k}$ & $0.64 \pm 0.03^{h}$ & $0.71 \pm 0.02^{\mathrm{de}}$ & $0.65 \pm 0.02^{g h}$ & $0.74 \pm 0.03^{c}$ \\
\hline 4 & $180(0)$ & $400(+1)$ & $20,000(0)$ & $60(-1)$ & $0.49 \pm 0.08^{\mathrm{op}}$ & $0.56 \pm 0.04^{\prime}$ & $0.62 \pm 0.01^{i}$ & $0.58 \pm 0.05^{k}$ & $0.65 \pm 0.04^{g h}$ \\
\hline 5 & $180(0)$ & $200(-1)$ & $16,000(-1)$ & $70(0)$ & $0.50 \pm 0.01^{\circ}$ & $0.56 \pm 0.05^{1}$ & $0.63 \pm 0.02^{\mathrm{hi}}$ & $0.57 \pm 0.06^{\mathrm{kl}}$ & $0.67 \pm 0.05^{\mathrm{fg}}$ \\
\hline 6 & $160(-1)$ & $300(0)$ & $24,000(+1)$ & $70(0)$ & $0.51 \pm 0.02^{\mathrm{no}}$ & $0.55 \pm 0.03^{\mathrm{lm}}$ & $0.62 \pm 0.01^{i}$ & $0.58 \pm 0.07^{k}$ & $0.68 \pm 0.04^{f}$ \\
\hline $7\left(C_{1}\right)$ & $180(0)$ & $300(0)$ & $20,000(0)$ & $70(0)$ & $0.53 \pm 0.02^{\mathrm{mn}}$ & $0.59 \pm 0.08^{\mathrm{jk}}$ & $0.66 \pm 0.05^{9}$ & $0.60 \pm 0.05^{j}$ & $0.69 \pm 0.08^{\mathrm{ef}}$ \\
\hline $8\left(C_{2}\right)$ & $180(0)$ & $300(0)$ & $20,000(0)$ & $70(0)$ & $0.53 \pm 0.04^{\mathrm{mn}}$ & $0.59 \pm 0.05^{j \mathrm{k}}$ & $0.66 \pm 0.06^{9}$ & $0.60 \pm 0.04^{j}$ & $0.69 \pm 0.06^{e f}$ \\
\hline 9 & $180(0)$ & $300(0)$ & $24,000(+1)$ & $60(-1)$ & $0.49 \pm 0.08^{\circ \mathrm{p}}$ & $0.55 \pm 0.04^{\mathrm{Im}}$ & $0.62 \pm 0.01^{i}$ & $0.56 \pm 0.05^{1}$ & $0.65 \pm 0.04^{g h}$ \\
\hline 10 & $200(+1)$ & $300(0)$ & $24,000(+1)$ & $70(0)$ & $0.60 \pm 0.05^{j}$ & $0.66 \pm 0.05^{9}$ & $0.73 \pm 0.02^{c d}$ & $0.67 \pm 0.06^{f g}$ & $0.76 \pm 0.05^{b}$ \\
\hline $11\left(C_{3}\right)$ & $180(0)$ & $300(0)$ & $20,000(0)$ & $70(0)$ & $0.53 \pm 0.09^{m n}$ & $0.59 \pm 0.07^{j \mathrm{k}}$ & $0.66 \pm 0.04^{9}$ & $0.60 \pm 0.03^{j}$ & $0.69 \pm 0.07^{\mathrm{ef}}$ \\
\hline $12\left(C_{4}\right)$ & $180(0)$ & $300(0)$ & $20,000(0)$ & $70(0)$ & $0.53 \pm 0.03^{m n}$ & $0.59 \pm 0.02^{j \mathrm{k}}$ & $0.66 \pm 0.07^{9}$ & $0.60 \pm 0.02^{j}$ & $0.69 \pm 0.05^{\mathrm{ef}}$ \\
\hline 13 & $180(0)$ & $200(-1)$ & $20,000(0)$ & $80(+1)$ & $0.58 \pm 0.01^{k}$ & $0.64 \pm 0.01^{h}$ & $0.71 \pm 0.02^{\text {de }}$ & $0.65 \pm 0.04^{g h}$ & $0.75 \pm 0.03^{b c}$ \\
\hline 14 & $200(+1)$ & $400(+1)$ & $20,000(0)$ & $70(0)$ & $0.60 \pm 0.05^{j}$ & $0.65 \pm 0.04^{g h}$ & $0.72 \pm 0.01^{d}$ & $0.67 \pm 0.06^{f g}$ & $0.76 \pm 0.05^{b}$ \\
\hline 15 & $160(-1)$ & $400(+1)$ & $20,000(0)$ & $70(0)$ & $0.50 \pm 0.04^{\circ}$ & $0.56 \pm 0.05^{1}$ & $0.63 \pm 0.02^{\mathrm{hi}}$ & $0.57 \pm 0.06^{\mathrm{kl}}$ & $0.66 \pm 0.05^{9}$ \\
\hline 16 & $180(0)$ & $400(+1)$ & $20,000(0)$ & $80(+1)$ & $0.62 \pm 0.01^{i}$ & $0.68 \pm 0.07^{f}$ & $0.75 \pm 0.04^{b c}$ & $0.67 \pm 0.06^{\mathrm{fg}}$ & $0.78 \pm 0.07^{\mathrm{ab}}$ \\
\hline 17 & $160(-1)$ & $300(0)$ & $20,000(0)$ & $80(+1)$ & $0.55 \pm 0.04^{\mathrm{Im}}$ & $0.61 \pm 0.05^{\mathrm{ij}}$ & $0.68 \pm 0.07^{f}$ & $0.62 \pm 0.01^{i}$ & $0.71 \pm 0.06^{\mathrm{de}}$ \\
\hline 18 & $200(+1)$ & $300(0)$ & $20,000(0)$ & $80(+1)$ & $0.65 \pm 0.04^{g h}$ & $0.71 \pm 0.03^{\mathrm{de}}$ & $0.78 \pm 0.06^{\mathrm{ab}}$ & $0.72 \pm 0.01^{d}$ & $0.81 \pm 0.02^{\mathrm{a}}$ \\
\hline 19 & $160(-1)$ & $200(-1)$ & $20,000(0)$ & $70(0)$ & $0.46 \pm 0.05^{\mathrm{pq}}$ & $0.52 \pm 0.04^{n}$ & $0.59 \pm 0.08^{\mathrm{jk}}$ & $0.54 \pm 0.08^{m}$ & $0.63 \pm 0.01^{\mathrm{hi}}$ \\
\hline 20 & $180(0)$ & $400(+1)$ & $16,000(-1)$ & $70(0)$ & $0.53 \pm 0.02^{\mathrm{mn}}$ & $0.59 \pm 0.01^{\mathrm{jk}}$ & $0.66 \pm 0.05^{9}$ & $0.60 \pm 0.04^{j}$ & $0.69 \pm 0.08^{\mathrm{ef}}$ \\
\hline 21 & $180(0)$ & $300(0)$ & $24,000(+1)$ & $80(+1)$ & $0.62 \pm 0.01^{i}$ & $0.68 \pm 0.02^{f}$ & $0.75 \pm 0.04^{b c}$ & $0.69 \pm 0.08^{e f}$ & $0.78 \pm 0.07^{\mathrm{ab}}$ \\
\hline 22 & $180(0)$ & $300(0)$ & $16,000(-1)$ & $60(-1)$ & $0.45 \pm 0.04^{9}$ & $0.51 \pm 0.03^{\text {no }}$ & $0.58 \pm 0.07^{k}$ & $0.52 \pm 0.04^{n}$ & $0.61 \pm 0.02^{i j}$ \\
\hline 23 & $180(0)$ & $400(+1)$ & $24,000(+1)$ & $70(0)$ & $0.57 \pm 0.06^{\mathrm{kl}}$ & $0.63 \pm 0.05^{\mathrm{hi}}$ & $0.70 \pm 0.06^{\mathrm{e}}$ & $0.54 \pm 0.03^{m}$ & $0.63 \pm 0.02^{h i}$ \\
\hline 24 & $160(-1)$ & $300(0)$ & $20,000(0)$ & $60(-1)$ & $0.42 \pm 0.01^{r}$ & $0.48 \pm 0.02^{p}$ & $0.55 \pm 0.01^{1 \mathrm{~m}}$ & $0.50 \pm 0.08^{\circ}$ & $0.59 \pm 0.07^{\mathrm{jk}}$ \\
\hline $25\left(C_{5}\right)$ & $180(0)$ & $300(0)$ & $20,000(0)$ & $70(0)$ & $0.53 \pm 0.02^{m n}$ & $0.59 \pm 0.04^{j k}$ & $0.66 \pm 0.02^{9}$ & $0.60 \pm 0.01^{j}$ & $0.69 \pm 0.04^{e f}$ \\
\hline 26 & $200(+1)$ & $300(0)$ & $16,000(-1)$ & $70(0)$ & $0.56 \pm 0.05^{1}$ & $0.62 \pm 0.04^{i}$ & $0.69 \pm 0.03^{\mathrm{ef}}$ & $0.63 \pm 0.02^{\mathrm{hi}}$ & $0.72 \pm 0.01^{d}$ \\
\hline 27 & $180(0)$ & $200(-1)$ & $24,000(+1)$ & $70(0)$ & $0.53 \pm 0.02^{\mathrm{mn}}$ & $0.59 \pm 0.01^{j \mathrm{k}}$ & $0.67 \pm 0.02^{\mathrm{fg}}$ & $0.60 \pm 0.04^{j}$ & $0.69 \pm 0.08^{e f}$ \\
\hline 28 & $200(+1)$ & $200(-1)$ & $20,000(0)$ & $70(0)$ & $0.56 \pm 0.05^{\prime}$ & $0.62 \pm 0.04^{i}$ & $0.69 \pm 0.08^{\mathrm{ef}}$ & $0.63 \pm 0.02^{h i}$ & $0.72 \pm 0.01^{d}$ \\
\hline 29 & $200(+1)$ & $300(0)$ & $20,000(0)$ & $60(-1)$ & $0.52 \pm 0.01^{n}$ & $0.58 \pm 0.02^{k}$ & $0.65 \pm 0.04^{g h}$ & $0.69 \pm 0.08^{\mathrm{ef}}$ & $0.74 \pm 0.03^{c}$ \\
\hline
\end{tabular}

$\overline{C 1, C 2, C 3, C 4, C 5}$ represent spraying drying process at center points

Experimental model $=$ Box-Behnken design

Total number of spray drying treatments $=29$

No of replicates $=03$

a-r values with similar letters show homogenous group within row and column $(p>0.05)$

samples were higher $(0.78 \pm 0.06,0.81 \pm 0.02 \mathrm{meq} / \mathrm{kg}$ $\mathrm{O}_{2}$ ) when compared to initial readings 0 day $(0.65 \pm$ $0.04 \mathrm{meq} / \mathrm{kg} \mathrm{O} \mathrm{O}_{2}$ ).

The regression equations regarding the response factors at different days and storage intervals after spray drying process have been summarized in the Table 9. The optimized conditions of inlet air temperature (161$\left.162{ }^{\circ} \mathrm{C}\right)$, feed flow rate $(310-320 \mathrm{~mL} / \mathrm{hr})$, atomization speed (16550-16,600 rpm) and outlet air temperature $\left(61-62^{\circ} \mathrm{C}\right)$ were found for maximum retention of fatty acids at $25^{\circ} \mathrm{C}$ after 60 days as ALA (123-124 mg/50 g egg), EPA (6.3-6.4 mg/50 g egg), DHA (11.6-11.8 mg/50 $\mathrm{g}$ egg), total omega-3 fatty acids $(141-142 \mathrm{mg} / 50 \mathrm{~g}$ egg) and PV $\left(0.60-0.61 \mathrm{meq} / \mathrm{kg} \quad \mathrm{O}_{2}\right)$ of DEDP samples, respectively.

Several authors, in accordance with our results, report the effect of storage temperature on egg powder fatty acids composition. Deslypere et al. [24] results also conclude that storage at lower temperatures for several months yielded no perceptible changes in $n-3$ PUFAs of 
Table 9 Regression equations for response factors at different days and storage intervals after spray drying process

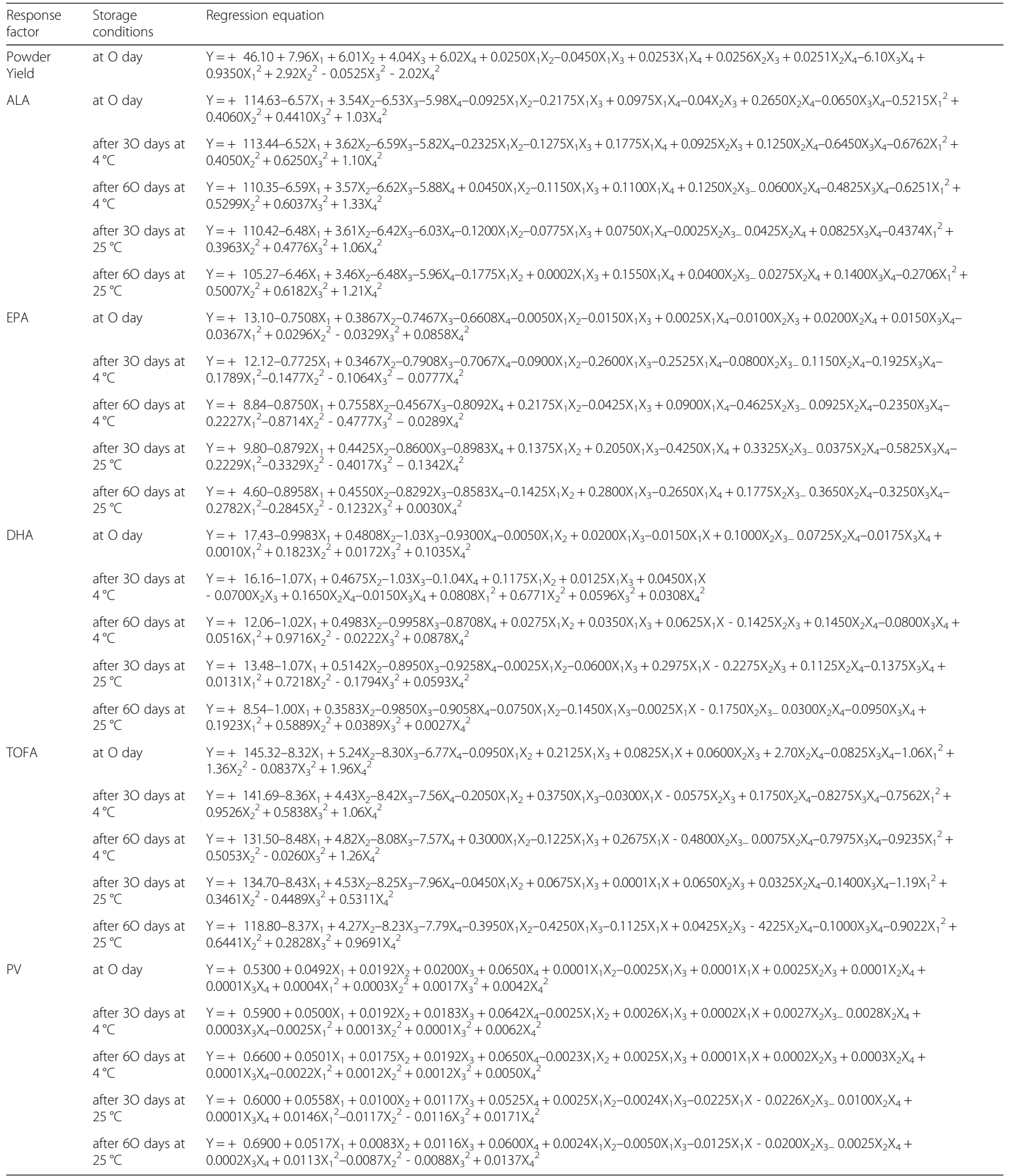

$\mathrm{X}_{1}=$ Inlet air temperature; $\mathrm{X}_{2}=$ Feed flow rate; $\mathrm{X}_{3}=$ Atomization speed; $\mathrm{X}_{4}=$ Outlet air temperature ALA Alpha-linolenic fatty acids, EPA Eicosapentaenoic fatty acids, DHA Docosahexaenoic fatty acids, TOFA Total omega-3 fatty acids, PV Peroxide value

fat tissue aspirates which is compatible with our results. This study showed that PUFAs loses increases with storage which is in accordance with observation of Terao et al. [25] that egg lipids underwent high oxidation during spray-drying; moreover, they observed that this oxidation significantly increases during storage (1 and 3 
months). Furthermore, several previous research studies described monounsaturated fatty acid and PUFAs losses during extensive heat processing [26-28]. In addition, some studies have been focused only on n-3 PUFAs losses because of their high nutritional relevance [29].

In the case of DEDP, the loss of essential fatty acids was already predicted because the PUFA ${ }_{S}$ content were high in DEDP samples and the heat treatment applied was severe. The thermal effects could be clearly observed at high outlet air temperatures, in accordance with other published reports. With increase of temperature, retention of PUFAs decreases and browning of powder increased, but lower temperature cause retention of moisture and low-quality powder with increased drying time [30, 31]. High temperature treatment causes protein denaturation and modifies lipoprotein structure. This change leads to decreased oxidative stability of egg lipids [32]. Higher temperature conditions in spray dryer causes higher losses of omega- 6 and omega-3 PUFAs and also less favorable to omega-6/omega-3 and PUFA/SFA ratios. Mostly $\mathrm{C}_{20: 4 \mathrm{n}-6}$ and $\mathrm{C}_{22: 6 \mathrm{n}-3}$ PUFAs are destroyed at high temperature [33].

The safety and quality of powdered eggs depend on at least two critical steps as the drying process itself and the storage conditions such as length and temperature. The drying process uses high temperatures that can accelerate reactions between lipids and molecular oxygen, resulting in losses of nutritional and sensory properties of egg products. At the same time, there is an increasing interest on the consumption of food that have a higher content of omega-3 PUFAs than conventional foods. However, this increase in the unsaturation of fatty acid can lead to an increase of lipid oxidation, especially during the drying process or the storage.

Unsaturated fatty acid losses have been widely reported as an indicator of lipid oxidation. As a rule, in foods, susceptibility to oxidation of phospholipids increases with the unsaturation [34]. The spray-dried eggs are highly oxidized and very susceptible to oxidation in comparison with raw eggs [35]. This fact is related to the structure of phospholipids in the raw yolk that protect against oxidation. Phospholipids are interwoven in the exterior structure of low-density lipoprotein and this compact surface prevents the contact of oxygen with the lipid core of the particle [36].

Egg powder was produced under high temperature scales, which led to many changes in egg components, resulting in lower retention of PUFAs in DEDP samples during storage. Food industries of using spray dried omega food materials are facing the problem of oxidation as these possessed unstable PUFAs during processing and storage. Several appropriate methods have been applied to reduce or prevent lipid oxidation of spray dried powders in order to improve final functional food quality. The most commonly used method is the addition of antioxidants. A combination of antioxidants with inert gas packaging can strongly stabilize the spray dried omega food products. Major finding supports that spray drying of whole egg at moderate conditions of air inlet temperature, feed flow rate, atomization speed and outlet air temperature resulted in a product that enhanced considerably the retention of PUFAs and good quality powder that could further be used for development of functional food products. Thereby, it could be concluded that slight lipid oxidation mostly occurs during spray-drying but this oxidation rate may be enhanced during storage. So, care should be taken during storage of DEDP samples.

\section{Conclusion}

The results of present study demonstrated the optimized conditions of inlet air temperature $\left(198-199^{\circ} \mathrm{C}\right)$, feed flow rate $(398-399 \mathrm{~mL} / \mathrm{hr})$, atomization speed $(16000$ $16,010 \mathrm{rpm})$ and outlet air temperature $\left(76-77^{\circ} \mathrm{C}\right)$ for maximum yield of designer egg dried powder samples $(66.20 \pm 0.20 \mathrm{~g} / 500 \mathrm{~mL})$. The inlet and outlet air temperature were seen to be as major factors affecting the essential fatty acids content in spray dried samples. Furthermore, the results from this work will aid in the formulation of healthy food products supplemented with designer egg dried powder and may address a critical industrial demand in terms of formulation options. Additional studies should be undertaken to enhance the shelf life of omega food products by supplementation of antioxidants and gradual reduction of oxidation process. Furthermore, future studies should focus on treatment of nutritional disorders through the functional foods and their absorption, metabolism and distribution pattern into biological tissues.

\section{Acknowledgements}

The authors are highly obliged to the Library Department, Government College University Faisalabad (GCUF) and IT Department, Higher Education Commission (HEC, Islamabad) for access to journals, books and valuable database.

\section{Funding \\ The authors are highly obliged to the Department of Food Science, Nutrition and Home Economics for providing chemicals to carried out the analysis of the samples.}

\section{Availability of data and materials}

The dataset supporting the conclusions of this article is included within the article.

\section{Authors' contributions}

AJ conceptualized, performed, analyzed and MI provided the technical assistance while NA and AlH guided for drafting the manuscript. "It's also confirmed that all the authors read and approved the final manuscript".

Ethics approval and consent to participate Not Applicable. 


\section{Consent for publication}

Not Applicable.

\section{Competing interests}

The authors declare that they have no competing interests.

\section{Publisher's Note}

Springer Nature remains neutral with regard to jurisdictional claims in published maps and institutional affiliations.

\section{Author details}

'Department of Food Science, Nutrition and Home Economics, Government College University, Faisalabad, Pakistan. ${ }^{2}$ Institute of Home and Food Sciences, Faculty of Life Sciences, Government College University, Faisalabad, Pakistan. ${ }^{3}$ Department of Chemistry, Faculty of Physical Sciences, Government College University, Faisalabad, Pakistan.

Received: 4 October 2018 Accepted: 26 November 2018

Published online: 13 December 2018

\section{References}

1. Klurfeld DM. In: Akoh CC, Min DB, editors. Food lipids. 6th ed. New York: Marcel Dekker, Inc.; 2002. p. 589-602.

2. Attia YA, Al-Harthi MA, Korish MM, Shiboob MM. Fatty acid and cholesterol profiles, hypocholesterolemic, atherogenic, and thrombogenic indices of broiler meat in the retail market. Lipids Health Dis. 2017;16(40):1-11.

3. Shahidi F, Miraliakbari H. Omega-3 fatty acids in health and disease: part 2-health effects of omega-3 fatty acids in autoimmune diseases, mental health, and gene expression. J Med Food. 2005;82(2):133-48.

4. Shahidi F, Finley JW. Omega-3 fatty acids: chemistry, nutrition and health effects. Washington, D.C.: ACS Symposium Series, American Chemical Society; 2001. p. 303

5. Shahidi F, Miraliakbari H. Tree nut oils and byproducts: compositional characteristics and Nutra-ceutical applications. In: Nutraceutical and specialty lipids and their co-products. Boca Raton: CRC Press, Taylor \& Francis Group; 2006. p. 159-68.

6. Surai PF, Sparks NHC. Designer eggs: from improvement of egg composition to functional food. Trends Food Sci Technol. 2001:12(1):7-16.

7. Let MB, Jacobsen C, Meyer AS. Sensory stability and oxidation of fish oil enriched milk is affected by milk storage temperatures and oil quality. Int Dairy J. 2005;15(2):173-82.

8. Kruidenier L, Verspaget HW. Review article: oxidative stress as a pathogenic factor in inflammatory bowel disease--radicals or ridiculous? Aliment Pharmacol Ther. 2002;16(12):1997-2015

9. Davies KJ. Oxidative stress, antioxidant defenses, and damage removal, repair, and replacement systems. IUBMB Life. 2000;50(4-5):279-89.

10. Floyd RA, Hensley K. Oxidative stress in brain aging. Implications for therapeutics of neurodegenerative diseases. Neurobiol Aging. 2002;23(5): 795-807.

11. Schuck P, Dolivet A, Méjean S, Zhu P, Blanchard E, Jeantet R. Drying by desorption: a tool to determine spray drying parameters. J Food Eng. 2009; 94(2):199-204

12. Koç B, Yılmazer MS, Balkir P, Ertekin FK. Spray drying of yoghurt: optimization of process conditions for improving viability and other quality attributes. Dry Technol. 2010;28(4):495-507.

13. Liu CP, Liu SD. Low-temperature spray drying for the microencapsulation of the fungus Beauveria bassiana. Dry Technol. 2009;27(6):747-53.

14. Re Ml. Formulating drug delivery systems by spray drying. Dry Technol. 2006;24(4):433-46.

15. Obon JM, Castellar MR, Alacid M, Fernandez-Lopez JA. Production of a redpurple food colorant from Opuntia stricta fruits by spray drying and its application in food model systems. J Food Eng. 2009;90(4):471-9.

16. Sagar VR, Suresh Kumar P. Recent advances in drying and dehydration of fruits and vegetables: a review. J Food Sci Technol. 2010;47(1):15-26.

17. Kumaravel S, Hema R, Kamaleshwari A. Effect of oven drying on the nutritional properties of whole egg and its components. Int J Food Sci Nutr. 2011:1(1):4-12.

18. Fernandez-perez V, Tapiador J, Martin A, luque de-castro MD. Optimization of drying step for preparing a new commercial powdered soup. Innov Food Sci Emerg Technol. 2004;5(3):361-8.
19. Bahnasawy AH, Okasha AE, Gonbeej EE. Performance evaluation of a laboratory scale spray dryer. Misr J Ag Eng. 2010;27(1):326-46.

20. AOAC. Official methods of analysis of the Association of Official Analytical Chemists. 15th ed. Arlington: Association of Official Analytical Chemists; 1990. p. 1058-9.

21. AOCS. Official methods and recommended practices of AOCS. 5th ed Champaign: American Oil Chemists Society; 1998.

22. Montgomery DC. Response surface methods and designs. In: Design and analysis of experiments. New York: Wiley Publishers; 2008. p. 440.

23. Ronald CD. Spray drying innovative use of an old process. J Des Elem. 1997; 7:97-113.

24. Deslypere JP, van de Bovenkamp P, Harryvan JL, Katan MB. Stability of n-3 fatty acids in human fat tissue aspirates during storage. Am J Clin Nutr. 1993;57(6):884-8.

25. Terao J, Kawanishi M, Matsushita S. Application of high-performance liquid chromatography for the estimation of peroxidized phospholipids in spraydried egg and muscle foods. J Agric Food Chem. 1987;35(4):613-7.

26. Dearden SJ, Hunter TF, Philp J. Fatty acid analysis as a function of photooxidation in egg yolk lecithin vesicles. Photochem Photobiol. 1985;41(2):213-5.

27. Gokalp HY, Ockerman HW, Plimpton RF, Harper WJ. Fatty acids of neutral and phospholipids, rancidity scores and TBA values as influenced by packaging and storage. J Food Sci. 1983:48(3):829-34.

28. Yan PS, White PJ. Linalyl acetate and methyl silicone effects on cholesterol and triglyceride oxidation in heated lard. J Am Oil Chem Soc. 1991;68(10):763-8.

29. Ajuyah AO, Ahn DU, Hardin RT, Sim JS. Dietary antioxidants and storage affect chemical characteristics of $\omega$-3 fatty acid enriched broiler chicken meats. J Food Sci. 1993;58(1):43-61.

30. Hardy J, Parmenties M, Fanni J. Functionality of nutrients and thermal treatments of food. Proc Nutr Soc. 1999;58(3):579-85.

31. Thomas ME, Scher J, Desobry-Banon S, Desobry S. Milk powders ageing: effect on physical and functional properties. Crit Rev Food Sci Nutr. 2004; 44(5):297-322.

32. Pike OA, Peng IC. Stability of shell egg and liquid yolk to lipid oxidation. Poult Sci. 1985;64(8):1470-5.

33. Guardiola F, Codony R, Manich A, Rafecas M, Boatella J. Stability of polyunsaturated fatty acids in egg powder processed and stored under various conditions. J Agric Food Chem. 1995:43(8):2254-9.

34. Pikul J, Kummerow FA. Thiobarbituric acid reactive substance formation as affected by distribution of polyenoic fatty acids in individual phospholipids. J Agric Food Chem. 1991;39(3):451-7.

35. Marshall AC, Sams AR, Van Elswyk ME. Oxidative stability and sensory quality of stored eggs from hens fed 1.5\% menhaden oil. J Food Sci. 1994;59(3): 561-3.

36. Burley RW, Vadehra DV. Egg yolk: structure and properties. In: The avian egg: chemistry and biology. New York: Wiley Publishers; 1989. p. 472.

Ready to submit your research? Choose BMC and benefit from:

- fast, convenient online submission

- thorough peer review by experienced researchers in your field

- rapid publication on acceptance

- support for research data, including large and complex data types

- gold Open Access which fosters wider collaboration and increased citations

- maximum visibility for your research: over $100 \mathrm{M}$ website views per year

At BMC, research is always in progress.

Learn more biomedcentral.com/submission 